Article

\title{
An Adaptive Sliding Mode Variable Admittance Control Method for Lower Limb Rehabilitation Exoskeleton Robot
}

\author{
Yao Tu ${ }^{1,2}$, Aibin Zhu ${ }^{1,2, *}$, Jiyuan Song ${ }^{1,2} \oplus$, Huang Shen ${ }^{1,3}$, Zhitao Shen ${ }^{1,3}$, \\ Xiaodong Zhang ${ }^{1,2}$ and Guangzhong Cao ${ }^{4}$ D \\ 1 Institute of Robotics \& Intelligent Systems, Xi'an Jiaotong University, Xi'an 710049, China; \\ tu1007909971@stu.xjtu.edu.cn (Y.T.); jysong@stu.xjtu.edu.cn (J.S.); shenhuang@stu.xjtu.edu.cn (H.S.); \\ shen940123@stu.xjtu.edu.cn (Z.S); xdzhang@mail.xjtu.edu.cn (X.Z.) \\ 2 Shaanxi Key Laboratory of Intelligent Robots, Xi'an 710049, China \\ 3 Key Laboratory of Education Ministry for Modern Design and Rotor-Bearing System, Xi'an 710049, China \\ 4 Guangdong Key Laboratory of Electromagnetic Control and Intelligent Robots, Shenzhen University, \\ Shenzhen 518060, China; gzcao@szu.edu.cn \\ * Correspondence: abzhu@mail.xjtu.edu.cn
}

Received: 14 February 2020; Accepted: 3 April 2020; Published: 7 April 2020

\begin{abstract}
As passive rehabilitation training with fixed trajectory ignores the active participation of patients, in order to increase the active participation of patients and improve the effect of rehabilitation training, this paper proposes an innovative adaptive sliding mode variable admittance (ASMVA) controller for the Lower Limb Rehabilitation Exoskeleton Robot. The ASMVA controller consists of an outer loop with variable admittance controller and an inner loop with an adaptive sliding mode controller. It estimates the wearer's active muscle strength and movement intention by judging the deviation between the actual and standard interaction force of the wearer's leg and the exoskeleton, thereby adaptively changing admittance controller parameters to alter training intensity. Three healthy volunteers engaged in further experimental studies, including trajectory tracking experiments with no admittance, fixed admittance, and variable admittance adjustment. The experimental results show that the proposed ASMVA control scheme has high control accuracy. Besides, the ASMVA can not only increase training intensity according to the active muscle strength of the patient during positive movement intention (so as to increase active participation of the patient), but also increase the amount of trajectory adjustment during negative movement intention to ensure the safety of the patient.
\end{abstract}

Keywords: exoskeleton; rehabilitation training; adaptive sliding mode control; variable admittance control

\section{Introduction}

With increase in the global aging population, the number of patients with lower limb dyskinesia caused by diseases such as locomotive syndrome and sarcopenia is on the rise. According to research on medical theory and clinical experience, patients with locomotive syndrome and sarcopenia can prevent muscle atrophy and promote muscle functional recovery through high-intensity and repetitive exercise rehabilitation training [1]. The lower limb rehabilitation exoskeleton (LLRE) robot is a human mechatronic system that integrates the robot and the wearer [2]. By simulating normal human walking gait, it helps patients with lower limb motor dysfunction to exercise lower limb muscles and restore function [3,4]. In the study of LLRE, especially in control strategies and methods, researchers focus 
more on patients' active participation in rehabilitation training [5] and safety and comfort during rehabilitation training [6].

For patients with lower limb paralysis, because the lower limb muscles do not actively contract [7], in order to prevent lower limb muscle atrophy, the affected limb is passively driven by the exoskeleton, and the purpose of limb movement recovery is gradually achieved under passive rehabilitation training. In the existing literature, various LLREs that can be used for passive rehabilitation training have been developed and studied, such as MINDWALKER [8] (University of Twente, Enschede, Netherlands), ATLAS [9] (Centre for Automation and Robotics, La Poveda, Madrid, Spain), ReWalk [10] (Rewalk Robotics, Marlborough, MA, USA), EKSO [11] (Ekso Bionics, Richmond, VA, USA), Twin [12] (IIT, Via Morego, Genova, Italy), etc. The control system of the above LLREs is essentially a passive position control system, because active participation of the wearer is not considered in the system. However, for patients with lower limb dysfunction, such as locomotive syndrome and sarcopenia, they still have a certain amount of muscle strength retained in their affected limbs [13]. The patient's active movement intention should be taken into consideration in rehabilitation training to improve active participation, thereby improving the effect of rehabilitation training [14].

In the study of LLRE control strategies for the rehabilitation training of patients with residual muscle strength in their affected limbs, two main issues need attention. The first is how exoskeleton adaptively adjusts training tasks according to the condition of different patients' affected lower limbs [15-17]. The second is how to optimize the exoskeleton control strategy based on the active muscle force of the patients' lower limbs, so that they can participate in rehabilitation training as much as possible to achieve maximum exercise of the muscles. The LLRE robot can directly interact with patients' lower limbs $[18,19]$. On one hand, LLRE needs to provide power for lower limbs to follow the standard gait trajectory [20]. On the other hand, it is necessary to ensure that the power provided can be adaptively adjusted according to the patient's active muscle force to ensure active participation [21].

In order to increase a patient's active participation in exoskeleton-assisted rehabilitation training, it is necessary to control the exoskeleton robot to track the movement of the lower limb as per the patient's active motion intention [22,23]. Impedance controller or admittance controller have been widely used in exoskeleton control due to their characteristics of human and robot physical interaction [24,25]. The impedance controller calculates the force exerted by the robot to achieve the specified displacement. Admittance control is based on the impedance control and uses an external force loop and an internal position loop to form the entire controller [26]. Aydin et al. [27] adopted an admittance controller to convert the interaction force between the human body and the exoskeleton into the expected trajectory of the exoskeleton, so as to recognize and track the operator's intention. Aiming at the safety of robot-assisted interactive motion of the affected limb, Pan et al. [28,29] proposed an exoskeleton impedance control method based on safety monitoring, but the active muscle force of the patient's limbs was not considered in human-robot interaction. Aguirre-ollinger et al. [30] analyzed the stability conditions of admittance control of a single-degree-of-freedom exoskeleton swinging leg, and believed that admittance control can improve the compliance of the exoskeleton leg [31]. Wang et al. [32] applied admittance control to the active training stage of patient rehabilitation, and adjusted the admittance parameters to meet the different needs for strength and speed during training. Morbi et al. [33] proposed that non-linear admittance control can promote a safer human-computer interaction in haptic devices. The above research results show that admittance control can effectively recognize the movement intention of exoskeleton wearers, and the control system using admittance control has a simple structure and is easy to implement. However, the above studies on exoskeleton admittance control have adopted fixed admittance parameters to control exoskeleton, which has limitations in the application of LLREs. On the one hand, when the patient has a negative intention, that is, the direction of the active torque of the lower limb joint is opposite to the preset motion trajectory speed, if the admittance parameters of the admittance controller is too large, that is, the exoskeleton stiffness is too large, the physical interaction between the patient's lower limbs and exoskeleton will increase, 
bringing discomfort and safety risks to the patient. On the other hand, when the patient has a positive intention, that is, the direction of the active torque of the lower limb joint is the same as the preset movement trajectory speed, if the admittance parameters of the admittance controller is too small, that is, the stiffness of the exoskeleton is too small, the intensity of the patient's rehabilitation training will decrease, leading to a decrease in active participation and thus a reduction in the rehabilitation effect.

For the rehabilitation training of patients with dyskinesia that have residual muscle strength in the affected limb, the passive position control scheme for LLRE ignores the active participation of patients in rehabilitation training and cannot fully meet the rehabilitation training needs of such kind of patients. Moreover, admittance control with fixed admittance parameters for LLRE ignores the patients' positive and negative intentions in rehabilitation training, and affects the comfort, safety and active participation of patients in rehabilitation training. Therefore, this paper described a novel ASMVA control strategy for LLRE, which can estimate the patients' movement intention based on the human-exoskeleton interaction force, and can actively adjust the intensity of rehabilitation training according to the patients' movement intention, which can effectively help patients exercise muscle to restore the muscle strength of the lower limbs, and solve the problems of passive rehabilitation training with fixed trajectory and admittance control with fixed admittance parameters.

\section{Exoskeleton System Overview}

\subsection{Hardware Description}

In order to carry out the task of lower limb rehabilitation training, LLRE was designed according to the anatomy of the lower limbs of the human body. Figure 1 shows the main structure of the lower extremity rehabilitation exoskeleton robot and a schematic diagram of the connection between the wearer and the exoskeleton. The LLRE has three motion joints on one leg, namely the hip joint, knee-joint, and ankle joint. There is an active degree of freedom (DOF) at the hip joint to make the thigh flexion/extension. The knee-joint has an active DOF to make the shank flexion/extension. In addition, the ankle joint has a passive DOF to make the foot flexion/extension. When a patient uses this exoskeleton, the patient's lower limbs need to be connected to the exoskeleton through a Velcro strap on the thighs and shanks of the exoskeleton.
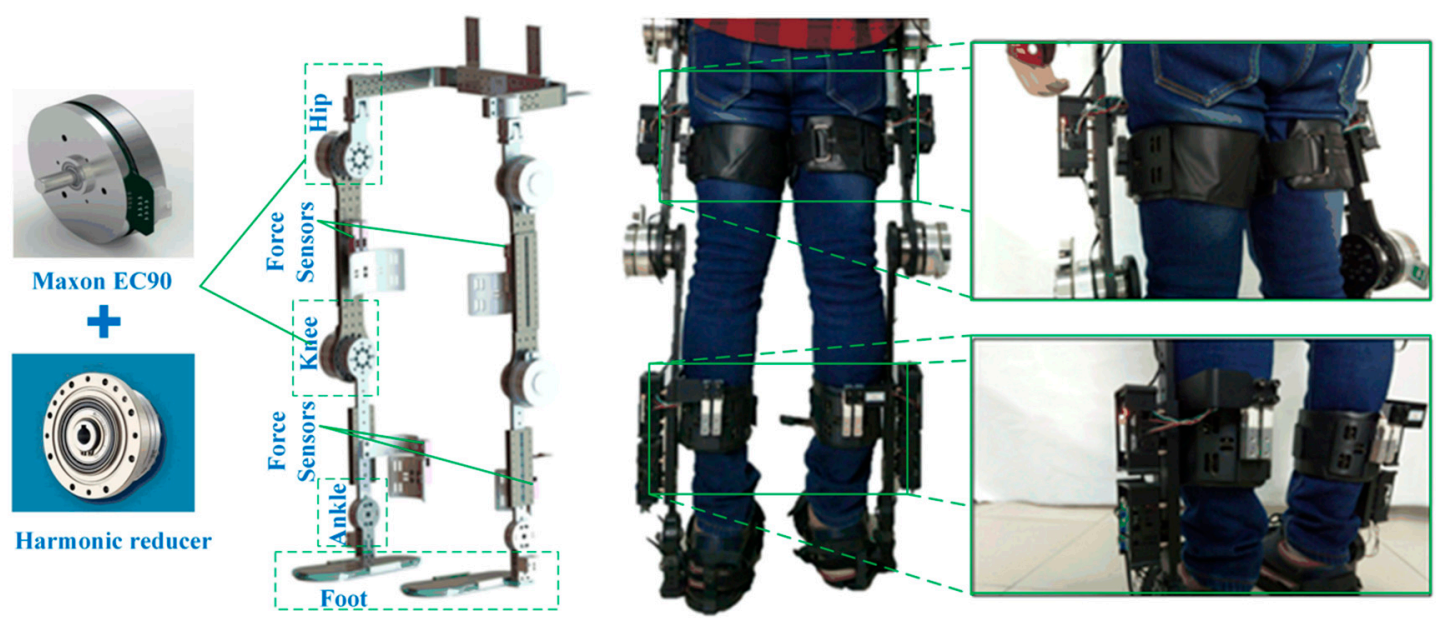

Figure 1. Main structure of LLRE and schematic diagram of the connection between the wearer and the exoskeleton.

The active DOFs of the LLRE robot need to be driven by brushless DC motors that have compact size and meet the required torque. The experimental platform of the LLRE robot uses the disk motor (Maxon EC90, maxon motor (Suzhou) Co., Suzhou, China) as the driving motor, which is a brushless DC motor with maximum speed of $2590 \mathrm{r} / \mathrm{min}$, rated current of $6.06 \mathrm{~A}$, and rated output torque of 
$0.444 \mathrm{~N} \cdot \mathrm{m}$. When it is used with a harmonic reducer at a reduction ratio of 160 , the rated output torque can theoretically reach $71 \mathrm{~N} \cdot \mathrm{m}$. The motor is also integrated with a 1024 lines rotary encoder, with 4096 pulses in one revolution of the motor, so the resolution of the rotary encoder to measure the rotation angle of the exoskeleton joint can theoretically reach 0.0005 degree.

The uniaxial force sensor (DSX-306, DESENTE, Shenzhen, China) is integrated into the LLRE robot to acquire real-time human-exoskeleton interaction force data during rehabilitation training. Two uniaxial force sensors are located on the connection between the bandage and the thigh and the shank of exoskeleton to measure the interaction force between the human lower limb and the exoskeleton, which is shown in Figure 2.

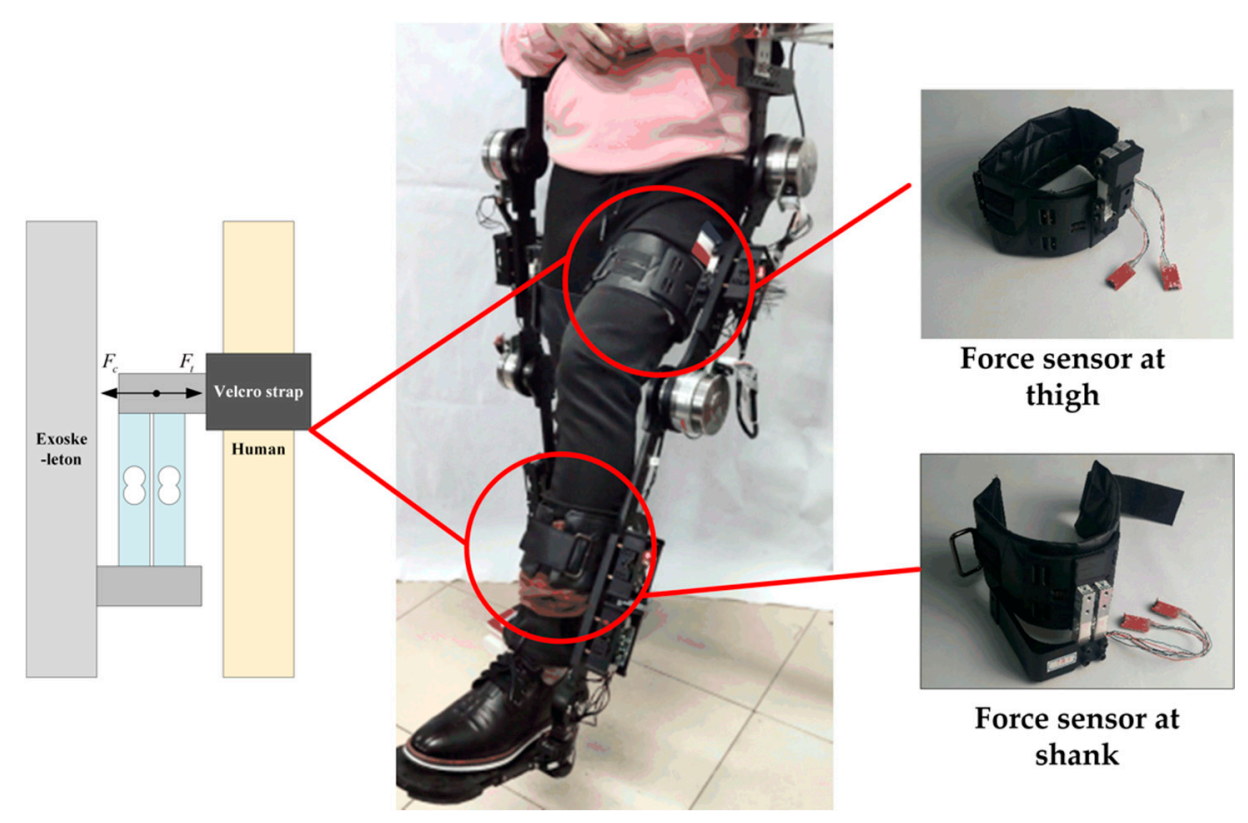

Figure 2. Force sensor installed between the exoskeleton and the human.

\subsection{Real-Time Control System}

The real-time control system of the LLRE robot is established in a Matlab/xPC environment (Mathworks, Inc., Natick, MA, USA) with a closed-loop hierarchical architecture, as shown in Figure 3. The control system includes the host controller and target controller; the host controller is a personal computer, and the target controller is a STM32F407 (STMicroelectronics, Geneva, Switzerland) embedded microcomputer. The host controller can generate the Simulink control model according to the preset control strategy, and then the control model can be converted into executable embedded control code and transmitted to the target controller through RS232 serial port in real time. The target controller can execute an embedded control code to realize operation of the LLRE robot. The force analog signal of the force sensor is collected by an HX711 (AVIA SEMICONDUCTOR (XIAMEN) Co., Xiamen, China) chip with high precision 24 bit AD converter and STM32F103 (STMicroelectronics, Geneva, Switzerland) embedded microcomputer. The motor driver is Copley-ACK-HC-ds-1 from Copley Controls Company (Peabody, MA, USA), whose power supply voltage is $24 \mathrm{~V}$, supporting speed and position; and current control modes, supporting Can-open communication mode. The Copley-ACK-HC-ds-1 driver can not only drive the Maxon EC90 motor, but also collect the data of the rotary encoder (maxon motor (Suzhou) Co., Suzhou, China) integrated in the motor. The collected data from force sensors and rotary encoders is transmitted to the target controller through the communication mode of Can-open, and the target controller also sends the control command to the motor driver through the communication mode of Can-open, whose communication rate can reach $1 \mathrm{Mbps} / \mathrm{s}$. 


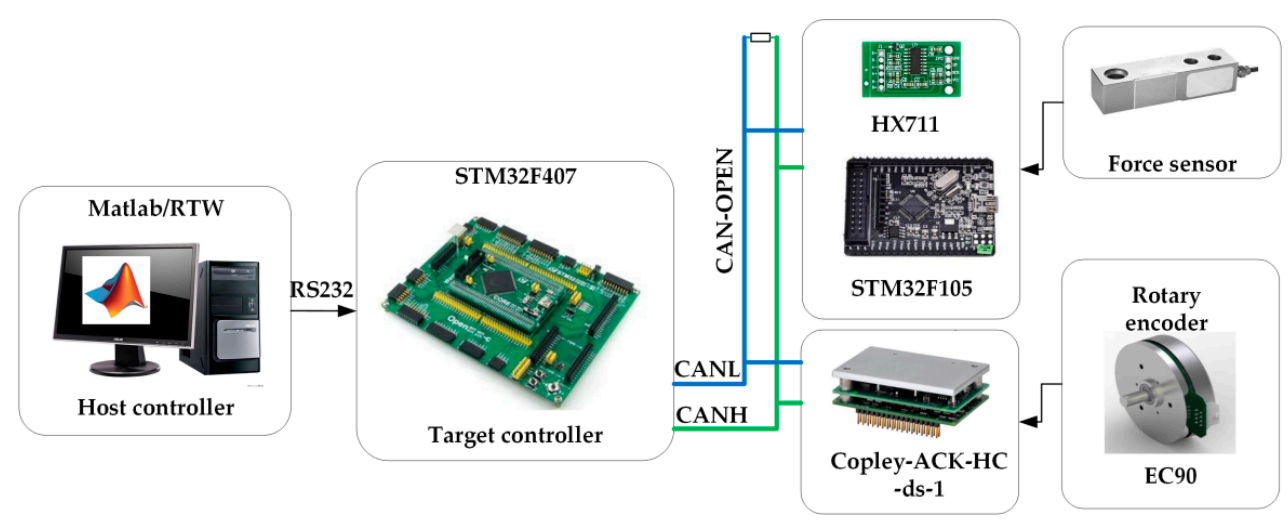

Figure 3. Real-time control system of the exoskeleton.

\section{Methods}

\subsection{Human-Exoskeleton Coupling Dynamics Modeling}

The exoskeleton robot has two unique characteristics, namely physical and cognitive interaction between human limbs and the exoskeleton. The physical interaction is a force interaction, and the exoskeleton can enhance the strength of human limbs. The cognitive interaction means that the wearer can feel that he is controlling the exoskeleton. At present, in the dynamic analysis of exoskeleton system, researchers usually only pay attention to the dynamic characteristics of the exoskeleton system itself, or take the exoskeleton system and human limb as a rigid connection for dynamic analysis, and ignore the physical interaction between the exoskeleton and the human limb. It is of great significance in the study of adaptive sliding mode variable admittance control of exoskeleton robot based on human-exoskeleton physical interaction to establish a coupling dynamic model of exoskeleton and human limb.

In this work, the 2-DOF connecting-rod model is adopted in coupling dynamics analysis of the exoskeleton and human limb. The 2-DOF connecting-rod model contains a trunk, thigh, and shank with the trunk locked, so the swing process is essentially the rotation of the thigh around the hip joint and the shank around the knee joint. Because the human leg is attached to the exoskeleton support, the muscle and fat in the human leg will produce soft coupling between the exoskeleton and the skeleton, which allows relative movement of the human leg skeleton and the exoskeleton. As shown in Figure $4 \mathrm{a}$, this soft coupling can be modeled using linear spring with coefficient $b_{i}$ and linear damper with coefficient $k_{i}$. The simplified model is shown in Figure $4 \mathrm{~b}$.

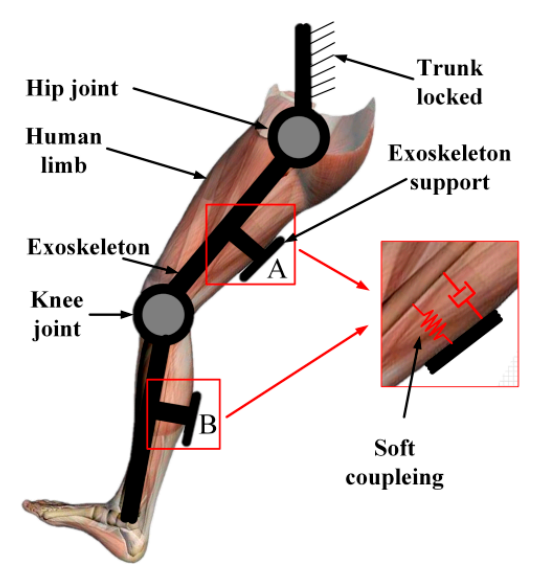

(a)

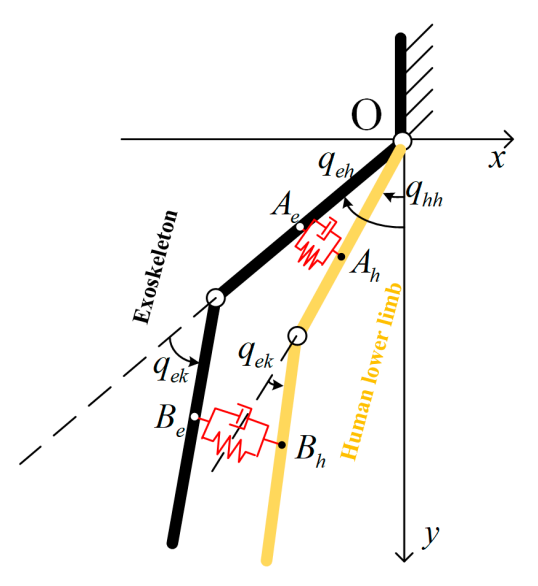

(b)

Figure 4. (a) The coupling model of human lower limb and exoskeleton. (b) The simplified model of the human lower limb (yellow) and the exoskeleton (black). 
According to the Euler-Lagrange equations, the dynamic model equation of a human leg can be described by:

$$
\mathbf{M}_{\mathbf{h}}\left(\mathbf{q}_{\mathbf{h}}\right) \ddot{\mathbf{q}}_{\mathbf{h}}+\mathbf{C}_{\mathbf{h}}\left(\mathbf{q}_{\mathbf{h}}, \dot{\mathbf{q}}_{\mathbf{h}}\right) \dot{\mathbf{q}}_{\mathbf{h}}+\mathbf{G}_{\mathbf{h}}\left(\mathbf{q}_{\mathbf{h}}\right)=\mathbf{T}_{\mathbf{h}}
$$

where $\mathbf{q}_{\mathbf{h}}=\left[q_{h h}, q_{h k}\right]^{T}$ denotes the state vector of human joint angle, $\dot{\mathbf{q}}_{\mathbf{h}}=\left[\dot{q}_{h h^{\prime}} \dot{q}_{h k}\right]^{T}$ denotes the state vector of human joint angular velocity, $\ddot{\mathbf{q}}_{\mathbf{h}}=\left[\ddot{q}_{h h}, \ddot{q}_{h k}\right]^{T}$ denotes the state vector of human joint angular acceleration. $\mathbf{T}_{\mathbf{h}}=\left[T_{h h}, T_{h k}\right]^{T}$ is the state vector of torque generated by human hip joint and knee joint. $\mathbf{M}_{\mathbf{h}} \in \mathbf{R}^{2 \times 2}$ is the inertia matrix of single human lower limb, which is a symmetric positive definite matrix. $\mathbf{C}_{\mathbf{h}} \in \mathbf{R}^{2}$ is the coriolis force vector. $\mathbf{G}_{\mathbf{h}} \in \mathbf{R}^{2}$ denotes the gravitational torque vector.

Similarly, the dynamic model equation of a 2-DOF exoskeleton is given as:

$$
\mathbf{M}_{\mathbf{e}}\left(\mathbf{q}_{\mathbf{e}}\right) \ddot{\mathbf{q}}_{\mathbf{e}}+\mathbf{C}_{\mathbf{e}}\left(\mathbf{q}_{\mathbf{e}}, \dot{\mathbf{q}}_{\mathbf{e}}\right) \dot{\mathbf{q}}_{\mathbf{e}}+\mathbf{G}_{\mathbf{e}}\left(\mathbf{q}_{\mathbf{e}}\right)=\mathbf{T}_{\mathbf{e}}
$$

where $\mathbf{q}_{\mathbf{e}}=\left[q_{e h}, q_{e k}\right]^{T}$ denotes the state vector of 2-DOF exoskeleton joint angle, $\dot{\mathbf{q}}_{\mathbf{e}}=\left[\dot{q}_{e h}, \dot{q}_{e k}\right]^{T}$ denotes the state vector of 2-DOF exoskeleton joint angular velocity, $\ddot{\mathbf{q}}_{\mathbf{e}}=\left[\ddot{q}_{e h}, \ddot{q}_{e k}\right]^{T}$ denotes the state vector of 2-DOF exoskeleton joint angular acceleration. $\mathbf{T}_{\mathbf{e}}=\left[T_{e h}, T_{e k}\right]^{T}$ is the vector of torque generated by the exoskeleton. $\mathbf{M}_{\mathbf{e}} \in \mathbf{R}^{2 \times 2}$ is the inertia matrix of exoskeleton, which is a symmetric positive definite matrix. $\mathbf{C}_{\mathbf{e}} \in \mathbf{R}^{2}$ is the coriolis force vector of the exoskeleton. $\mathbf{G}_{\mathbf{e}} \in \mathbf{R}^{2}$ denotes the gravitational torque vector of the exoskeleton.

There is a force interaction between the exoskeleton and the human lower limb. For patients with residual muscle strength in the lower limbs, their own muscle strength cannot help them walk normally, so the interaction force is a promotion for patients, but for the exoskeleton, the interaction force is a resistance, so the dynamic equation of human-exoskeleton coupling system can be given as:

$$
\left\{\begin{array}{l}
\mathbf{M}_{\mathbf{h}}\left(\mathbf{q}_{\mathbf{h}}\right) \ddot{\mathbf{q}}_{\mathbf{h}}+\mathbf{C}_{\mathbf{h}}\left(\mathbf{q}_{\mathbf{h}}, \dot{\mathbf{q}}_{\mathbf{h}}\right) \dot{\mathbf{q}}_{\mathbf{h}}+\mathbf{G}_{\mathbf{h}}\left(\mathbf{q}_{\mathbf{h}}\right)=\mathbf{T}_{\mathbf{h}}+\mathbf{J}_{\mathbf{h h}}{ }^{T} \mathbf{F}_{\mathbf{h}}+\mathbf{J}_{\mathbf{h k}}{ }^{T} \mathbf{F}_{\mathbf{k}} \\
\mathbf{M}_{\mathbf{e}}\left(\mathbf{q}_{\mathbf{e}}\right) \ddot{\mathbf{q}}_{\mathbf{e}}+\mathbf{C}_{\mathbf{e}}\left(\mathbf{q}_{\mathbf{e}}, \dot{\mathbf{q}}_{\mathbf{e}}\right) \dot{\mathbf{q}}_{\mathbf{e}}+\mathbf{G}_{\mathbf{e}}\left(\mathbf{q}_{\mathbf{e}}\right)=\mathbf{T}_{\mathbf{e}}-\mathrm{J}_{\mathbf{e h}}{ }^{T} \mathbf{F}_{\mathbf{h}}-\mathrm{J}_{\mathbf{e k}}{ }^{T} \mathbf{F}_{\mathbf{k}}
\end{array}\right.
$$

where $\mathbf{J}_{\mathbf{h h}}, \mathbf{J}_{\mathbf{h k}}$ are the Jacobian matrices of the human lower limb, and $\mathbf{J}_{\mathbf{e h}} \mathbf{J}_{\mathbf{e k}}$ are the Jacobian matrices of the 2-DOF exoskeleton. $\mathbf{F}_{\mathbf{h}}$ and $\mathbf{F}_{\mathbf{k}}$ denote the coupling terms of the human lower limb dynamics equation and 2-DOF exoskeleton dynamics equation, and their physical meanings are the interaction force of human limb and exoskeleton at the thigh and shank, respectively, and are given by:

$$
\begin{aligned}
& \mathbf{F}_{\mathbf{h}}=\left[\begin{array}{c}
F_{h x} \\
F_{h y}
\end{array}\right]=b_{i}\left[\begin{array}{c}
\dot{x}_{A e}-\dot{x}_{A h} \\
\dot{y}_{A e}-\dot{y}_{A h}
\end{array}\right]+k_{i}\left[\begin{array}{l}
x_{A e}-x_{A h} \\
y_{A e}-y_{A h}
\end{array}\right], \\
& \mathbf{F}_{\mathbf{k}}=\left[\begin{array}{c}
F_{h x} \\
F_{h y}
\end{array}\right]=b_{i}\left[\begin{array}{l}
\dot{x}_{B e}-\dot{x}_{B h} \\
\dot{y}_{B e}-\dot{y}_{B h}
\end{array}\right]+k_{i}\left[\begin{array}{l}
x_{B e}-x_{B h} \\
y_{B e}-y_{B h}
\end{array}\right],
\end{aligned}
$$

where $\left(x_{A e}, y_{A e}\right),\left(x_{A h}, y_{A h}\right)$ are coordinates of exoskeleton contact point $A_{e}$ and bone contact point $A_{h}$ at the connection of the exoskeleton and the thigh. $\left(x_{B e}, y_{B e}\right),\left(x_{B h}, y_{B h}\right)$ are coordinates of exoskeleton contact point $B_{e}$ and bone contact point $B_{h}$ at the connection of the exoskeleton and the shank. The coordinates of four points and their first derivative can be expressed as follows:

$$
\begin{gathered}
\left\{\begin{array}{l}
x_{A h}=l_{A h} \cos q_{h h} \\
y_{A h}=l_{A h} \sin q_{h h}
\end{array},\left\{\begin{array}{c}
x_{B h}=l_{h h} \cos q_{h h}+l_{B h} \cos \left(q_{h h}+q_{h k}\right) \\
y_{B h}=l_{h h} \sin q_{h h}+l_{B h} \sin \left(q_{h h}+q_{h k}\right)
\end{array}\right.\right. \\
\left\{\begin{array}{l}
x_{A e}=l_{A e} \cos q_{e h} \\
y_{A e}=l_{A e} \sin q_{e h}
\end{array},\left\{\begin{array}{l}
x_{B e}=l_{e h} \cos q_{e h}+l_{B e} \cos \left(q_{e h}+q_{e k}\right) \\
y_{B e}=l_{e h} \sin q_{e h}+l_{B e} \sin \left(q_{e h}+q_{e k}\right)
\end{array}\right.\right. \\
\left\{\begin{array}{c}
\dot{x}_{A h}=-l_{A h} \dot{q}_{h h} \sin q_{h h} \\
\dot{y}_{A h}=l_{A h} \dot{q}_{h h} \cos q_{h h}
\end{array},\left\{\begin{array}{l}
\dot{x}_{B h}=-l_{h h} \dot{q}_{h h} \sin q_{h h}-l_{B h}\left(\dot{q}_{h h}+\dot{q}_{h k}\right) \sin \left(q_{h h}+q_{h k}\right) \\
\dot{y}_{B h}=l_{h h} \dot{q}_{h h} \cos q_{h h}+l_{B h}\left(\dot{q}_{h h}+\dot{q}_{h k}\right) \cos \left(q_{h h}+q_{h k}\right)
\end{array}\right.\right.
\end{gathered}
$$




$$
\left\{\begin{array}{c}
\dot{x}_{A e}=-l_{A e} \dot{q}_{e h} \sin q_{e h} \\
\dot{y}_{A e}=l_{A e} \dot{q}_{e h} \cos q_{e h}
\end{array},\left\{\begin{array}{c}
\dot{x}_{B e}=-l_{e h} \dot{q}_{e h} \sin q_{e h}-l_{B e}\left(\dot{q}_{e h}+\dot{q}_{e k}\right) \sin \left(q_{e h}+q_{e k}\right) \\
\dot{y}_{B e}=l_{e h} \dot{q}_{e h} \cos q_{e h}+l_{B e}\left(\dot{q}_{e h}+\dot{q}_{e k}\right) \cos \left(q_{e h}+q_{e k}\right)
\end{array}\right.\right.
$$

where $l_{h h}$ and $l_{h k}$ denote lengths of the thigh and the shank of the human lower limb, $l_{e h}$ and $l_{e k}$ represent lengths of the thigh and the shank of the exoskeleton. It is assumed that the rotation axes of the first joint of exoskeleton and the human hip joint are coincident. Then $l_{A h}$ and $l_{B h}$ denote distance from the human hip joint and the knee joint to point $A_{h}$ and point $B_{h}, l_{A e}$ and $l_{B e}$ represent distance from the first joint and the second joint of exoskeleton to point $A_{e}$ and point $B_{e}$.

According to Figure 5, we can see that if the torque generated by the human is zero, then the human lower limb is driven entirely by the exoskeleton, and $T_{i}$ is the only driving force of the human lower limb, which we can call standard interaction torque $T_{i d}$. However, if the human lower limb retains some muscle strength, it means that the torque generated by the human is not zero. As a result, $T_{i}$ and $T_{h}$ are the driving forces of the human lower limb. Therefore, assuming that the trajectory of the exoskeleton joint is the same in both cases, if the torque generated by the human joint follows the rotation direction of the exoskeleton joint, the interaction torque between the human lower limb and the exoskeleton will be reduced, but if the torque generated by human joint does not follow the rotation direction of the exoskeleton joint, the interaction torque between the human lower limb and the exoskeleton will be increased. As a result, from the change of interaction torque, the torque generated by the human joint can be obtained, which shows motion intention. However, there is no good sensor measuring torque generated by human joint directly, but the interaction force between human lower limbs and exoskeleton can be measured easily by using a force sensor described above. According to interaction force, we can estimate the interaction torque between the human lower limbs and the exoskeleton. Finally, we can estimate the voluntary torque generated by the human joint by comparing the interactive torque with standard interactive torque. As shown in the equations below:

$$
\begin{gathered}
\mathbf{M}_{\mathbf{h}}\left(\mathbf{q}_{\mathbf{h}}\right) \ddot{\mathbf{q}}_{\mathbf{h}}+\mathbf{C}_{\mathbf{h}}\left(\mathbf{q}_{\mathbf{h}}, \dot{\mathbf{q}}_{\mathbf{h}}\right) \dot{\mathbf{q}}_{\mathbf{h}}+\mathbf{G}_{\mathbf{h}}\left(\mathbf{q}_{\mathbf{h}}\right)=\mathbf{T}_{\mathbf{i d}}, \\
\widetilde{\mathbf{T}}_{\mathbf{h}}=\mathbf{T}_{\mathbf{i d}}-\mathbf{J}_{\mathbf{h h}}{ }^{T} \mathbf{F}_{\mathbf{h}}-\mathbf{J}_{\mathbf{h k}}{ }^{T} \mathbf{F}_{\mathbf{k}},
\end{gathered}
$$

where $\mathbf{T}_{\mathbf{i d}}=\left[T_{i d h}, T_{i d k}\right]^{T}$ is the standard interactive torque, $\widetilde{\mathbf{T}}_{\mathbf{h}}$ is the estimating value of voluntary torque generated only by the wearer himself, $\widetilde{\mathbf{T}}_{\mathbf{h}}$ can be obtained by standard interactive torque $\mathbf{T}_{\mathbf{i d}}$ subtracting the actual measured interactive torque $\mathbf{J}_{\mathbf{h h}}{ }^{T} \mathbf{F}_{\mathbf{h}}+\mathbf{J}_{\mathbf{h k}}{ }^{T} \mathbf{F}_{\mathbf{k}}$.

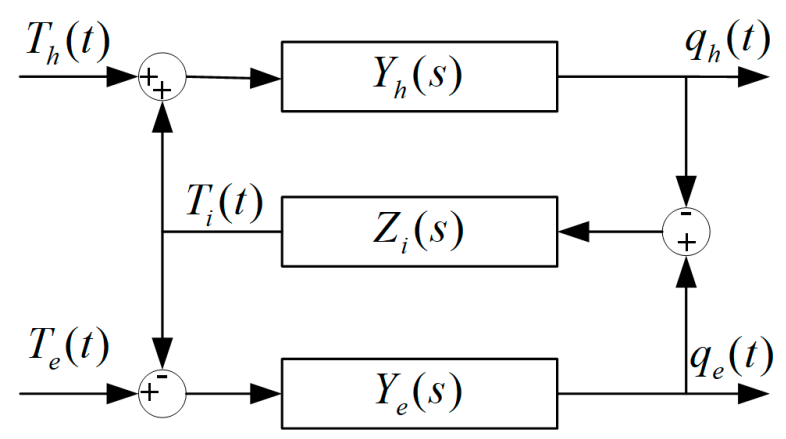

Figure 5. Expression of impedance and admittance of coupling model of the human lower limb and the exoskeleton.

\subsection{Variable Admittance Controller Design}

In humans, the brain is the controller, and the muscle is the actuator. In normal walking, the main problem of patients with lower muscle strength is that their muscles cannot provide the required torque to make them walk normally. In other words, when the human leg is in a swing phase, the torque provided by the muscles is not enough to make the human leg swing to the desired angle. Therefore, an admittance controller is proposed, which can judge human motion intention according to the interaction force between the human and the exoskeleton, and control the human exoskeleton system to make 
the human leg swing to the desired angle. The admittance control structure of the human-exoskeleton coupling system is shown in Figure 6. It is worth noting that the movement is still determined by the wearer, and the admittance controller adjusts the reference rehabilitation training trajectory to provide appropriate assistance according to the wearer's motion intention.

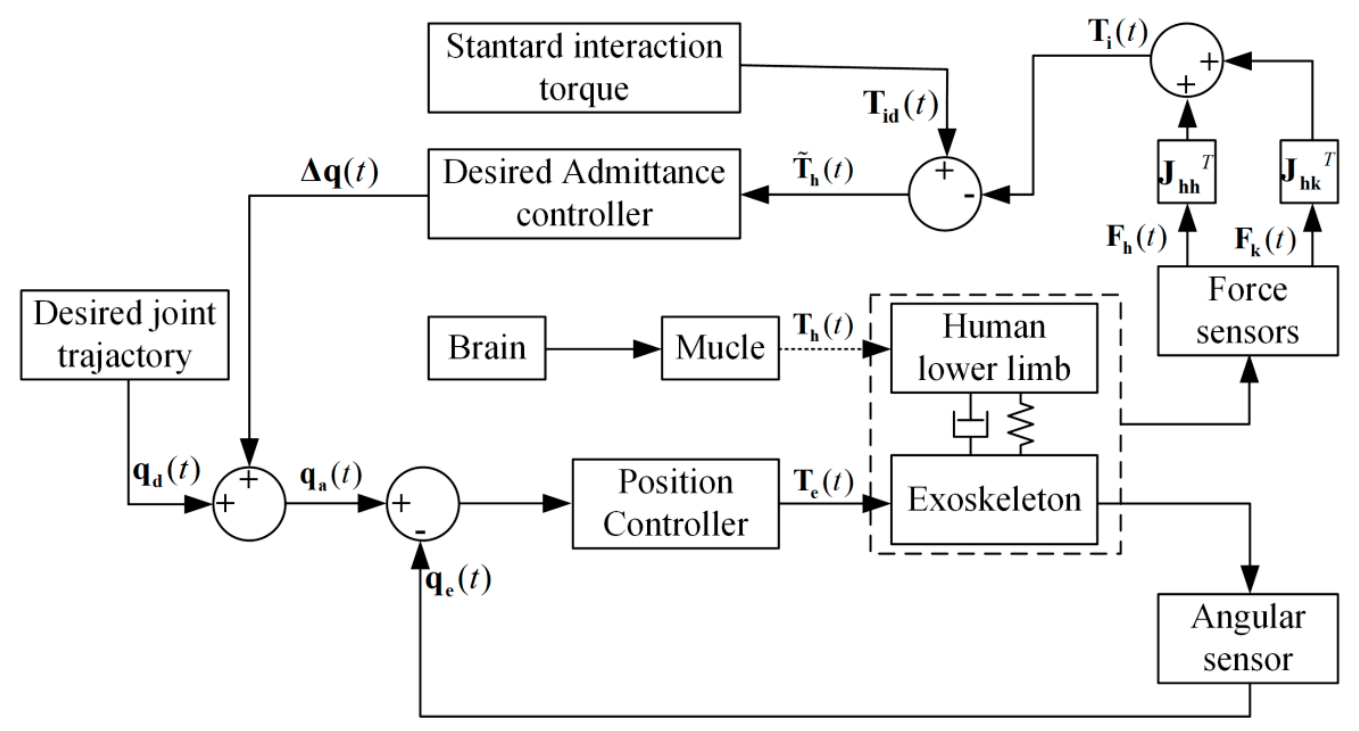

Figure 6. Admittance control structure of the human exoskeleton coupling system.

Admittance controller is the reciprocal variant of the impedance controller, whose performance depends on the accuracy of the force sensor and the position sensor. Compared with impedance control, admittance control is usually easier to implement in hardware. According to the definition of impedance, the desired impedance model of interaction between the human lower limbs and the exoskeleton can be designed as:

$$
\left\{\begin{array}{l}
\widetilde{\mathbf{T}}_{\mathbf{h}}(t)=\mathbf{J}_{\mathbf{d}} \Delta \ddot{\mathbf{q}}(t)+\mathbf{B}_{\mathbf{d}} \Delta \dot{\mathbf{q}}(t)+\mathbf{K}_{\mathbf{d}} \Delta \mathbf{q}(t) \\
\Delta \mathbf{q}(t)=\mathbf{q}_{\mathbf{a}}(t)-\mathbf{q}_{\mathbf{d}}(t)
\end{array}\right.
$$

where $\mathbf{J}_{\mathbf{d}}, \mathbf{B}_{\mathbf{d}}$, and $\mathbf{K}_{\mathbf{d}}$ are desired rotary inertia matrix, desired rotary damping matrix and desired rotary stiffness matrix respectively; $\mathbf{q}_{\mathbf{d}}$ and $\mathbf{q}_{\mathbf{a}}$ are desired move trajectory vector and desired control trajectory vector of the exoskeleton for rehabilitation of human lower limb in joint space, so $\Delta \mathbf{q}$ is the correction of the desired trajectory; $\widetilde{\mathbf{T}}_{\mathbf{h}}$ is the estimated voluntary torque generated by the human joint, which can be obtained by the above method.

Because the patient's own muscle strength is small, the patient's movement is relatively slow in the rehabilitation training. Thus, the acceleration term in the impedance model could be ignored; by only considering the rotation damping coefficient and rotation stiffness coefficient, the following impedance model could be obtained:

$$
\left\{\begin{array}{l}
\widetilde{\mathbf{T}}_{\mathbf{h}}(t)=\mathbf{B}_{\mathbf{d}} \Delta \dot{\mathbf{q}}(t)+\mathbf{K}_{\mathbf{d}} \Delta \mathbf{q}(t) \\
\Delta \mathbf{q}(t)=\mathbf{q}_{\mathbf{a}}(t)-\mathbf{q}_{\mathbf{d}}(t)
\end{array},\right.
$$

In the frequency domain space of the control system of exoskeleton, Equation (13) can be rephrased as:

$$
Y(s)=\frac{\Delta \mathbf{Q}(s)}{\widetilde{\mathbf{T}}_{\mathbf{h}}(s)}=\frac{1}{\mathbf{B}_{\mathbf{d}} s+\mathbf{K}_{\mathbf{d}}},
$$

In the process of rehabilitation training, the rotational stiffness and damping of the human lower limb joint will change according to the voluntary torque of the human; therefore, the parameters of the admittance controller also need to be changed according to changes in human voluntary 
torque. Based on the voluntary torque of the wearer's lower limb joint, the admittance parameters are changed. When the voluntary torque of the human joint is large, the parameters of the admittance controller should be appropriately increased to increase the training intensity and active participation of the wearer. When the voluntary torque of the human joint is small, the parameters of the admittance controller should be appropriately reduced to make it easier for the wearer to move the exoskeleton.

In rehabilitation training, the patients' intention of movement can be divided into two types: one is that the lower limbs voluntarily follow the desired trajectory, the other is that the lower limbs resist following the desired trajectory, which can be shown as follows for the single joint:

$$
\begin{aligned}
& \text { Intention }=\left\{\begin{array}{l}
\text { Positive movement } \operatorname{sign}\left(\widetilde{T}_{h}(t)\right)=\operatorname{sign}\left(\dot{q}_{d}(t)\right) \\
\text { Negtive movement } \operatorname{sign}\left(\widetilde{T}_{h}(t)\right) \neq \operatorname{sign}\left(\dot{q}_{d}(t)\right)
\end{array},\right. \\
& B_{v}=\left\{\begin{array}{c}
B_{p 0}+\left|\frac{\widetilde{T}_{h}(t)}{T_{i d}(t)}\right| \alpha_{p}, \text { for positive movement } \\
B_{n 0}-\left|\frac{\widetilde{T}_{h}(t)}{T_{i d}(t)}\right| \alpha_{n}, \text { for negative movement }
\end{array},\right. \\
& K_{v}=\left\{\begin{array}{l}
K_{p 0}+\left|\frac{\widetilde{T}_{h}(t)}{T_{i d}(t)}\right| \beta_{p}, \text { for positive movement } \\
K_{n 0}-\left|\frac{\widetilde{T}_{h}(t)}{T_{i d}(t)}\right| \beta_{n}, \text { for negative movement }
\end{array},\right.
\end{aligned}
$$

where $B_{v}$ and $K_{v}$ are actively used damping and stiffness, respectively, $B_{p 0}$ and $B_{n 0}$ are base damping for positive moment and negative movement, respectively, $K_{p 0}$ and $K_{n 0}$ are base stiffness for positive movement and negative movement, respectively. The parameters $\alpha_{p}$ and $\alpha_{n}$ are tuned manually to control the rate of change of $B_{v}$, and the parameters $\beta_{p}$ and $\beta_{n}$ are tuned manually to control the rate of change of $K_{v}$.

The variable admittance controller, established based on the voluntary torque generated by the human lower limb, can adaptively adjust the admittance parameters according to the patients' movement intention, and modify the desired trajectory to obtain the control reference trajectory in joint space, which can not only ensure the active participation of the patients, but also reduce interaction to ensure training safety and comfort.

\subsection{Design of Adaptive Sliding Mode Controller}

When patients wear the exoskeleton for rehabilitation training, the lower limbs may have involuntary shaking, and the exoskeleton robot may also have uncertain vibration, which will cause disturbance to the exoskeleton robot system and affect the accuracy of the tracking reference control trajectory of the exoskeleton robot. Therefore, in this paper, a sliding mode control method based on RBF neural network is proposed for position control of the exoskeleton. This algorithm uses sliding mode control to enhance the robustness of the control system against uncertain disturbances, and RBF neural network to fit the system model, which is difficult to accurately model to reduce chattering of the sliding mode controller.

Considering the modeling error and uncertainty disturbance of the human-machine coupling dynamic model, the dynamic model of exoskeleton robot can be rewritten as:

$$
\mathbf{M}_{\mathbf{e}}\left(\mathbf{q}_{\mathbf{e}}\right) \ddot{\mathbf{q}}_{\mathbf{e}}+\mathbf{C}_{\mathbf{e}}\left(\mathbf{q}_{\mathbf{e}}, \dot{\mathbf{q}}_{\mathbf{e}}\right) \dot{\mathbf{q}}_{\mathbf{e}}+\mathbf{G}_{\mathbf{e}}\left(\mathbf{q}_{\mathbf{e}}\right)+\mathbf{J}_{\mathbf{e h}}{ }^{T} \mathbf{F}_{\mathbf{h}}+\mathbf{J}_{\mathbf{e k}}{ }^{T} \mathbf{F}_{\mathbf{k}}+\tau_{\mathbf{d}}=\mathbf{u}
$$

where $\mathbf{u}$ is the exoskeleton control torque, and $\tau_{\mathbf{d}}$ is the uncertainty caused by modeling error and system disturbance.

The goal of designing the sliding mode controller is to ensure that the actual trajectory $\mathbf{q}_{\mathbf{e}}(t)=$ $\left[q_{e h}(t), q_{e k}(t)\right]^{T}$ of exoskeleton can track the command trajectory $\mathbf{q}_{\mathbf{d}}(t)=\left[q_{d h}(t), q_{d k}(t)\right]^{T}$. It assumed 
that the trajectory tracking error is $\mathbf{e}(t)=\mathbf{q}_{\mathbf{d}}(t)-\mathbf{q}_{\mathbf{e}}(t)$, where $\mathbf{e}(t)=\left[e_{h}(t), e_{k}(t)\right]^{T}$. The sliding surface is designed as follows:

$$
\mathbf{r}(t)=\dot{\mathbf{e}}(t)+\Lambda \mathbf{e}(t)
$$

where $\Lambda=\operatorname{diag}\left(\Lambda_{1}, \Lambda_{2}\right)$ is a positive definite matrix, $\mathbf{r}(t)=\left[r_{1}(t), r_{2}(t)\right]^{T}$ is the slope of the sliding surface. Then:

$$
\begin{aligned}
& \mathbf{M}_{\mathbf{e}} \dot{\mathbf{r}}=\mathbf{M}_{\mathbf{e}}\left(\ddot{\mathbf{q}}_{\mathbf{d}}-\ddot{\mathbf{q}}+\Lambda \dot{\mathbf{e}}\right)=\mathbf{M}_{\mathbf{e}}\left(\ddot{\mathbf{q}}_{\mathbf{d}}+\Lambda \dot{\mathbf{e}}\right)-\mathbf{M}_{\mathbf{e}} \ddot{\mathbf{q}}=\mathbf{M}_{\mathbf{e}}\left(\ddot{\mathbf{q}}_{\mathbf{d}}+\Lambda \dot{\mathbf{e}}\right)-\mathbf{u}+\mathbf{C}_{\mathbf{e}} \dot{\mathbf{q}}_{\mathbf{e}}+\mathbf{G}_{\mathbf{e}}+\mathbf{J}_{\mathbf{e h}}{ }^{T} \mathbf{F}_{\mathbf{h}} \\
& +\mathbf{J}_{\mathbf{e k}}{ }^{T} \mathbf{F}_{\mathbf{k}}+\boldsymbol{\tau}_{\mathbf{d}}=\mathbf{M}_{\mathbf{e}}\left(\ddot{\mathbf{q}}_{\mathbf{d}}+\Lambda \dot{\mathbf{e}}\right)-\mathbf{C}_{\mathbf{e}} \mathbf{r}+\mathbf{C}_{\mathbf{e}}\left(\dot{\mathbf{q}}_{\mathbf{d}}+\Lambda \mathbf{e}\right)+\mathbf{G}_{\mathbf{e}}+\mathbf{J}_{\mathbf{e h}}{ }^{T} \mathbf{F}_{\mathbf{h}}+\mathbf{J}_{\mathbf{e k}}{ }^{T} \mathbf{F}_{\mathbf{k}}+\boldsymbol{\tau}_{\mathbf{d}}-\mathbf{u}= \\
& -\mathbf{u}+\mathbf{f}+\mathbf{J}_{\mathbf{e h}}{ }^{T} \mathbf{F}_{\mathbf{h}}+\mathbf{J}_{\mathbf{e k}}{ }^{T} \mathbf{F}_{\mathbf{k}}+\boldsymbol{\tau}_{\mathbf{d}}
\end{aligned}
$$

where $\mathbf{f}=\mathbf{M}_{\mathbf{e}}\left(\ddot{\mathbf{q}}_{\mathbf{d}}+\boldsymbol{\Lambda} \dot{\mathbf{e}}\right)-\mathbf{C}_{\mathbf{e}} \mathbf{r}+\mathbf{C}_{\mathbf{e}}\left(\dot{\mathbf{q}}_{\mathbf{d}}+\mathbf{\Lambda} \mathbf{e}\right)+\mathbf{G}_{\mathbf{e}}$ is the uncertainty term in the exoskeleton dynamics model. In engineering applications, the uncertain term $\mathbf{f}$ of the model is unknown, so it is necessary to approximate the uncertain term $\mathbf{f}$. The basic idea of the RBF neural network is to use the radial basis function as the basis of the hidden layer neurons to form the hidden layer space. The hidden layer transforms the input data of the low-dimensional mode into the high-dimensional space, which makes the linear indivisible problem in the low-dimensional space linearly separable in the high-dimensional space. The RBF neural network is simple in structure, simple in training, and fast in convergence, and can approach any nonlinear function; therefore, the RBF neural network is used to approximate the uncertain term $\mathbf{f}$.

Taking $\mathbf{x}=\left[\mathbf{e}^{T}, \dot{\mathbf{e}}^{T}, \mathbf{q}_{\mathbf{d}}{ }^{T}, \dot{\mathbf{q}}_{\mathbf{d}}{ }^{T}, \ddot{\mathbf{q}}_{\mathbf{d}}{ }^{T}\right]$ as the input of the RBF neural network, and taking Gaussian function as a membership function, the RBF neural network algorithm can be denoted as follows:

$$
\begin{gathered}
\mathbf{h}_{\mathbf{i}}=\exp \left(-\left\|\mathbf{x}-\mathbf{c}_{\mathbf{i}}\right\|^{2} / 2 \mathbf{b}_{\mathbf{i}}{ }^{2}\right), \\
\mathbf{f}(\mathbf{x})=\mathbf{W}^{T} \mathbf{h}(\mathbf{x})+\varepsilon,
\end{gathered}
$$

where $i=1,2,3, \cdots, m$, and $m$ is the number of neurons in the hidden layer of the RBF neural network, $\mathbf{c}_{\mathbf{i}}$ and $\mathbf{b}_{\mathbf{i}}$ are the center value and standardization constant of Gaussian function of the $i$-th neuron in the hidden layer of the RBF neural network, respectively, $\mathbf{W}$ is the connection weight matrix from the hidden layer to the output layer, $\varepsilon$ is the approximation error.

Due to the existence of approximation error, the actual output of the neural network can be set as:

$$
\hat{\mathbf{f}}(\mathbf{x})=\hat{\mathbf{W}}^{T} \mathbf{h}(\mathbf{x})
$$

where $\hat{\mathbf{W}}$ is the actual weight matrix of the RBF neural network when approaching $\mathbf{f}, \mathbf{h}(\mathbf{x})$ is the hidden layer basis vector of the RBF neural network.

The sliding mode control law is designed as follows:

$$
\mathbf{u}=\hat{\mathbf{W}}^{T} \mathbf{h}(\mathbf{x})+\mathbf{J}_{\mathbf{e h}}{ }^{T} \mathbf{F}_{\mathbf{h}}+\mathbf{J}_{\mathbf{e k}}{ }^{T} \mathbf{F}_{\mathbf{k}}+\mathbf{K r}+\mathbf{v},
$$

where $\mathbf{K}$ is the control parameter of the sliding mode controller, $\mathbf{r}$ is the designed sliding surface, $\boldsymbol{v}$ is the robust term of the controller to overcome system disturbance.

Considering the approximation error $\varepsilon$ of the neural network and the disturbance term $\tau_{\mathrm{d}}$ in the actual exoskeleton control, the robust term $v$ is designed as:

$$
\boldsymbol{v}=-\left(\varepsilon_{\mathbf{N}}+\mathbf{b}_{\mathbf{d}}\right) \operatorname{sgn}(\mathbf{r}),
$$

where $\varepsilon_{\mathbf{N}} \geq\|\mathbf{f}-\hat{\mathbf{f}}\|$ is the maximum permissible error of RBF neural network approximation, $\mathbf{b}_{\mathbf{d}} \geq$ $\left\|\mathbf{J}_{\mathbf{e h}}{ }^{T} \mathbf{F}_{\mathbf{h}}+\mathbf{J}_{\mathbf{e k}}{ }^{T} \mathbf{F}_{\mathbf{k}}+\boldsymbol{\tau}_{\mathbf{d}}\right\|$ is the maximum disturbance of the system. 


\subsection{Proof of System Stability}

The Lyapunov function of the control system is defined as follows:

$$
L=\frac{1}{2} \mathbf{r}^{T} \mathbf{M}_{\mathbf{e}} \mathbf{r}+\frac{1}{2} \operatorname{tr}\left(\widetilde{\mathbf{W}}^{T} \mathbf{F}^{-1} \widetilde{\mathbf{W}}\right),
$$

where $\mathbf{F}$ is a positive definite matrix, $\widetilde{\mathbf{W}}$ is the weight error matrix of the RBF neural network in approximation, $\operatorname{tr}(\mathbf{X})$ is to trace matrix $\mathbf{X}$, whose result is a constant value.

The first derivative of the Lyapunov function shown in Equation (26) is obtained as follows:

$$
\dot{L}=\mathbf{r}^{T} \mathbf{M}_{\mathbf{e}} \dot{\mathbf{r}}+\frac{1}{2} \mathbf{r}^{T} \dot{\mathbf{M}}_{\mathbf{e}} \mathbf{r}
$$

Combining Equation (20), Equation (22), and Equation (24):

$$
\dot{L}=-\mathbf{r}^{T} \mathbf{K r}+\mathbf{r}^{T}\left(\varepsilon+\mathbf{J}_{\mathbf{e h}}{ }^{T} \mathbf{F}_{\mathbf{h}}+\mathbf{J}_{\mathbf{e k}}{ }^{T} \mathbf{F}_{k}+\boldsymbol{\tau}_{\mathbf{d}}+\boldsymbol{v}\right),
$$

Due to:

$$
\begin{aligned}
& \mathbf{r}^{T}\left(\varepsilon+\mathbf{J}_{\mathbf{e h}}{ }^{T} \mathbf{F}_{\mathbf{h}}+\mathbf{J}_{\mathbf{e k}}{ }^{T} \mathbf{F}_{\mathbf{k}}+\boldsymbol{\tau}_{\mathbf{d}}+\boldsymbol{v}\right)=\mathbf{r}^{T}\left(\varepsilon+\mathbf{J}_{\mathbf{e h}}{ }^{T} \mathbf{F}_{\mathbf{h}}+\mathbf{J}_{\mathbf{e k}}{ }^{T} \mathbf{F}_{\mathbf{k}}+\boldsymbol{\tau}_{\mathbf{d}}\right)+\mathbf{r}^{T} \boldsymbol{v} \\
& =\mathbf{r}^{T}\left(\varepsilon+\mathbf{J}_{\mathbf{e h}}{ }^{T} \mathbf{F}_{\mathbf{h}}+\mathbf{J}_{\mathbf{e k}}{ }^{T} \mathbf{F}_{\mathbf{k}}+\boldsymbol{\tau}_{\mathbf{d}}\right)-\mathbf{r}^{T}\left(\varepsilon_{\mathbf{N}}+\mathbf{b}_{\mathbf{d}}\right) \leq 0
\end{aligned}
$$

Then $\dot{L} \leq 0$. According to Lyapunov's stability discrimination theorem, when $\mathbf{r} \neq 0, \dot{L}(\mathbf{r})<0$ and $\mathbf{r}=0, \dot{L}(\mathbf{r})=0$. Because $\dot{L}(\mathbf{r})$ is a negative definite, it is asymptotically stable at the origin, and because $\dot{L} \leq 0, \mathbf{r}$ and $\mathbf{e}$ is uniformly bounded by Lyapunov stability theory. In addition, because $\mathbf{r}=\dot{\mathbf{e}}+\Lambda \mathbf{e}, \dot{e}$ is uniformly bounded, therefore, according to Barbalat's lemma, when $t \rightarrow \infty, \dot{L}(\mathbf{r})=0$, it is $\mathbf{r} \rightarrow 0$, thus $\mathbf{e} \rightarrow 0$ and $\dot{\mathbf{e}} \rightarrow 0$, which proves that the control system is stable.

\subsection{Experimental Design and Implementation}

In order to verify the effectiveness of the ASMVA controller for the LLRE robot, the human-exoskeleton interaction measurement experiment and the track tracking experiments under different admittance conditions based on the exoskeleton platform were carried out in this paper, as shown in Figure 7. In these experiments, the subject's left leg was connected to the exoskeleton through a Velcro strap at the thigh and shank of the exoskeleton, and the exoskeleton was fixed on the base made of aluminum profiles by screws to avoid falling and shaking of the exoskeleton. During these experiments, the actual movement track of the exoskeleton joint was measured in real time by the rotary encoder, and the interaction torque of the knee joint was measured in real time by the interaction force sensor installed between the shank of subject and the shank of exoskeleton. The motor drivers were set as torque control mode, which provided auxiliary force for the lower limbs of the human body according to the control algorithm and the feedback sensor signal. Three healthy subjects with different body measurement parameters (H1: male, 24 years old, weight $/ 60 \mathrm{~kg}$, height $/ 1.70 \mathrm{~m} ; \mathrm{H} 2$ : male, 23 years old weight $/ 65 \mathrm{~kg}$, height $/ 1.75 \mathrm{~m}$, H3: female, 25 years old, weight $/ 51 \mathrm{~kg}$, height $/ 1.60 \mathrm{~m}$ ) participated in the study and provided consent before the experiment. This study was approved by the biomedical ethics committee of the medical department of $\mathrm{Xi}^{\prime}$ an Jiaotong University. 


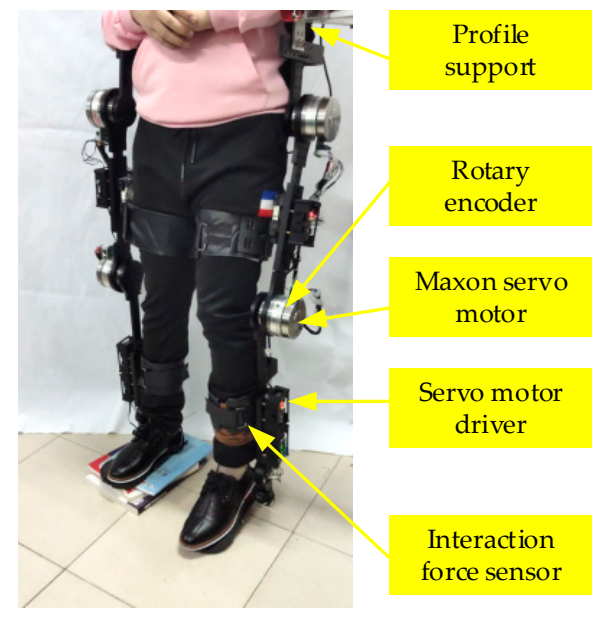

Figure 7. The LLRE robot prototype worn by a healthy subject.

\subsubsection{Human-Exoskeleton Interaction Measurement Experiment}

The purpose of the human-exoskeleton interaction measurement experiment was to obtain the relationship between the angular displacement and the standard interaction torque of the knee-joint. This experiment was based on the subject's knee-joint flexion movement, which is shown in Figure 8. During this experiment, the left leg of the subject did not generate any active force, and the exoskeleton adopted position mode control, that is, the joint of the exoskeleton rotated according to the preset motion track under the servo drive system. Firstly, the hip-joint of exoskeleton rotated by 35 degrees in flexion, then the hip-joint of exoskeleton locked up, and the knee-joint of the exoskeleton extended and flexed two times in a reciprocating motion; the joint rotation amplitude was 60 degrees. Finally, the hip-joint of the exoskeleton was rotated by 35 degrees in flexion motion to restore its vertical state.

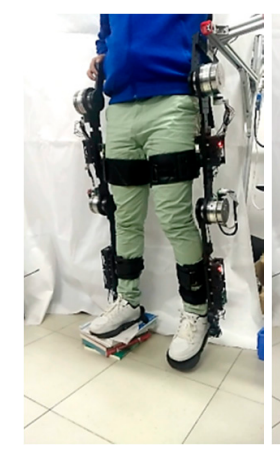

(a)

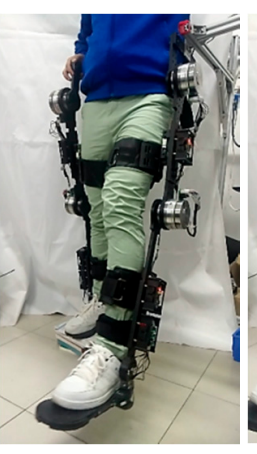

(b)

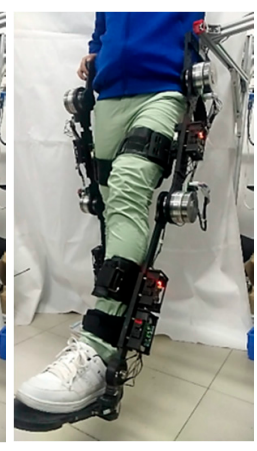

(c)

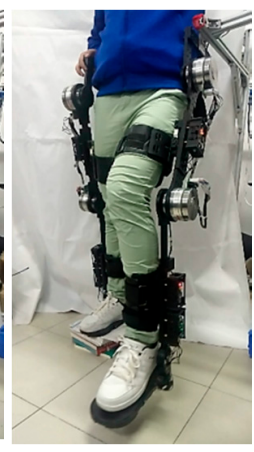

(d)

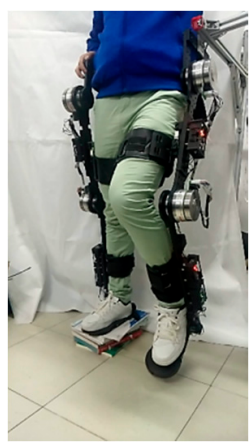

(e)

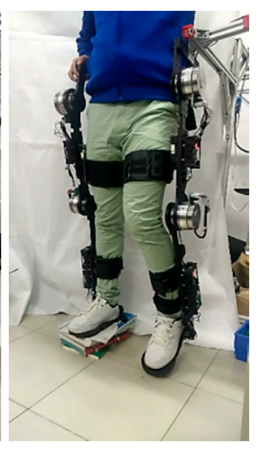

(f)

Figure 8. The experimental procedure. (a) Vertical state. (b) Hip-joint starts to flex. (c) Hip-joint rotates to 35 degrees. (d) Knee joint starts to extend. (e) Knee joint rotates to 60 degrees. (f) Exoskeleton restores to vertical state.

\subsubsection{Track Tracking Experiments Under Different Admittance Conditions}

The track tracking experiments under different admittance conditions mainly includes three sub-experiments, which are track tracking experiment with no admittance, track tracking experiment with fixed admittance, and track tracking experiment with variable admittance. Track tracking experiment with no admittance was carried out to verify the position control performance of the proposed ASMVA controller. Through the track tracking experiment with fixed admittance, the influence of different fixed admittance parameters on track tracking was analyzed. Track tracking 
experiment with variable admittance was carried out to verify that the proposed ASMVA controller could adjust admittance parameters according to patients' movement intention in training.

- No admittance

According to the overall block diagram of the control strategy shown in Figure 6, the inner loop ASMC (adaptive sliding mode controller) controller is responsible for accurate tracking of the preset trajectory of the knee-joint adjusted by the admittance controller. In fact, if the admittance parameters are all set to infinity, the ASMVA controller can be transformed into a position controller to track the predefined training track. Therefore, this track tracking experiment with no admittance was carried out to prove the control accuracy of the inner loop ASMC controller. The initial state of track tracking experiment was that the hip joint of exoskeleton drove the subject's leg to rotate 35 degrees, and then the hip joint was locked. During this experiment, the knee joint track was preset as a sine wave track with frequency of $0.1 \mathrm{~Hz}$, peak amplitude of 50 degrees and phase of $\pi / 2$. The time required for each experiment was $20 \mathrm{~s}$, that was, the subject's shank swung two cycles along the preset sine wave track driven by the exoskeleton. In order to ensure control accuracy and system stability, the parameters of the GSMC (general sliding mode controller) algorithm were tested many times and modulated optimally. The recommended control parameters for ASMVA controller were set to $\Lambda=50, K=55, \varepsilon_{N}=0.5, b_{d}=0.6, B_{p 0}=B_{n 0}=10^{10}, K_{p 0}=K_{n 0}=10^{10}, \alpha_{p}=\alpha_{n}=0, \beta_{p}=\beta_{n}=0$. The recommended configurations of the RBF neural network were: the number of hidden layer nodes was $7, \mathbf{c}=[-1.5,-1.0,-0.5,0,0.5,1.0,1.5], \mathbf{b}=[12,12,12,12,12,12,12]$.

\section{- Fixed admittance}

The purpose of the passive training mode with no admittance is to ensure the accuracy of repeated track tracking during rehabilitation training, and it is suitable for patients without any active muscle strength. However, if the patient restores part of muscle strength, the introduction of admittance control into passive training mode can improve the flexibility of human-exoskeleton interaction and ensure the comfort of the patient in the training process. In order to verify the influence of the ASMVA controller on track tracking under different fixed admittance parameters, subject H1 (male, 24 years old, weight $/ 60 \mathrm{~kg}$, height $/ 1.70 \mathrm{~m}$ ) was required to wear the exoskeleton for a track tracking experiment under three different admittance controller configurations of large admittance, medium admittance, and small admittance. During this experiment, the left leg of the subject was required to generate some active force, and the desired control trajectory was the same as that of track tracking experiment under infinite admittance condition; three different admittance controller configurations of this experiment are shown in Table 1.

Table 1. Three different admittance configurations.

\begin{tabular}{ccccc}
\hline $\begin{array}{c}\text { Admittance } \\
\text { Configurations }\end{array}$ & $\begin{array}{c}\boldsymbol{B}_{\boldsymbol{p} 0}, \boldsymbol{B}_{n 0} \\
\mathbf{( N m} \cdot \mathbf{s} / \text { degree })\end{array}$ & $\begin{array}{c}\boldsymbol{\alpha}_{\boldsymbol{p}}, \boldsymbol{\alpha}_{\boldsymbol{n}} \\
\mathbf{( N m} \cdot \mathbf{s} / \mathbf{d e g r e e})\end{array}$ & $\begin{array}{c}\boldsymbol{K}_{p 0}, \boldsymbol{K}_{n 0} \\
\mathbf{( N m} / \text { degree) }\end{array}$ & $\begin{array}{c}\boldsymbol{\beta}_{\boldsymbol{p} 0}, \boldsymbol{\beta}_{\boldsymbol{n} 0} \\
\mathbf{( N m} / \mathbf{d e g r e e})\end{array}$ \\
\hline Small admittance & 0.25 & 0 & 20 & 0 \\
Medium admittance & 0.5 & 0 & 40 & 0 \\
Large admittance & 1.0 & 0 & 80 & 0 \\
\hline
\end{tabular}

- Variable admittance

There are two situations in actual rehabilitation training of patients with residual muscle strength. One is that the direction of the knee joint active torque is the same as the speed direction of the desired training track, which can be regarded as the patient's positive movement intention. The other is that the direction of the knee joint active torque is opposite to the speed direction of the desired training track, which can be regarded as the patient's negative movement intention. In order to verify the performance of the ASMVA controller on the recognition of patients' movement intention and track tracking under 
the condition of variable admittance, subject H1 (male, 24 years old, weight $/ 60 \mathrm{~kg}$, height $/ 1.70 \mathrm{~m}$ ) was required to wear an exoskeleton for track tracking experiment under the condition of variable admittance. During this experiment, the left leg of the subject was required to generate some active force, and the desired control trajectory was the same as that of the track tracking experiment under infinite admittance condition; the variable admittance controller configurations of this experiment are shown in Table 2.

Table 2. Variable admittance configurations.

\begin{tabular}{ccccccc}
\hline Movement Intention & Range of $\boldsymbol{B}_{\boldsymbol{v}}$ & $\boldsymbol{B}_{0}$ & $\boldsymbol{\alpha}$ & Range of $\boldsymbol{K}_{\boldsymbol{v}}$ & $\boldsymbol{K}_{0}$ & $\boldsymbol{\beta}$ \\
\hline Positive & $\leq 1.5$ & 0.5 & 1.0 & $\leq 120$ & 40 & 30 \\
Negative & $\geq 0.2$ & 0.4 & 0.2 & $\geq 10$ & 35 & 15 \\
\hline
\end{tabular}

\section{Results}

\subsection{Human-Exoskeleton Interaction Measurement Experiment}

Figure 9 shows the results of subject $\mathrm{H} 1$ (male, 24 years old, weight $/ 60 \mathrm{~kg}$ ) in the human-exoskeleton interaction measurement experiment. The motion trajectories of the hip joint and knee joint are shown in Figure 9a,b, demonstrating the interaction torque of the knee joint, the positive direction of which is counterclockwise. The $5-25 \mathrm{~s}$ data is selected for further processing. The measured angular displacement of the knee joint is used as an independent variable, and the interaction torque of the knee joint is used as a dependent variable to make a graph in the same coordinate system, as shown in Figure 10. It can be seen that there is a linear relationship between angular displacement of the knee joint and standard interaction torque of the knee joint, which can be fitted by using the least square method. The fitted linear relationships of three subjects between the angular displacement of the knee joint and the standard interaction torque of the knee joint are shown in Table 3, where $y$ is the standard interaction torque of the knee joint, and $x$ is the angular displacement of the knee joint, whose range is 0 degrees to 60 degrees.

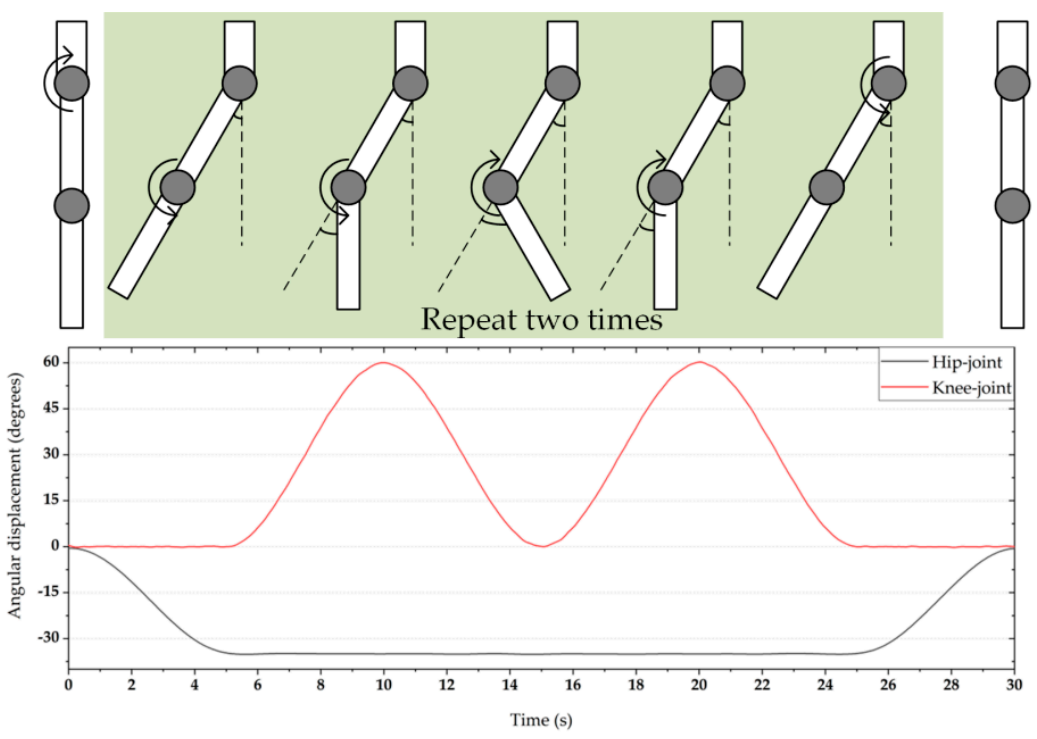

(a)

Figure 9. Cont. 


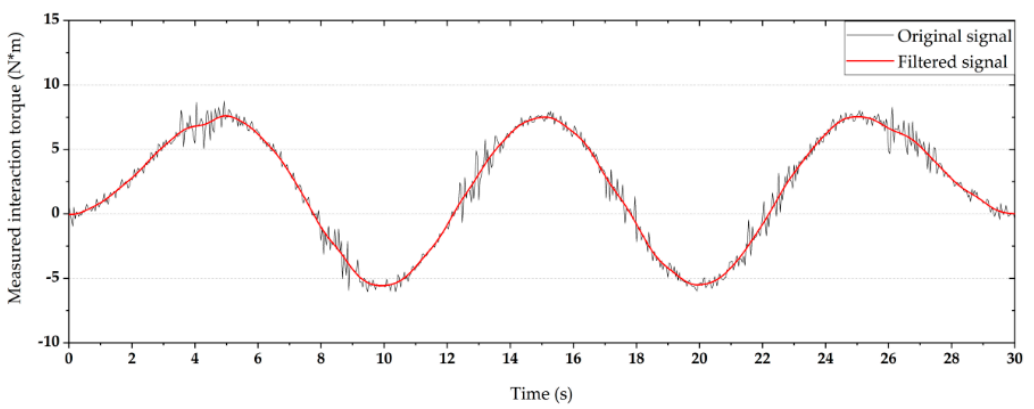

(b)

Figure 9. Measured data. (a) Motion trajectory of the hip joint (black line) and knee joint (red line). (b) Measured interaction torque of the knee joint.

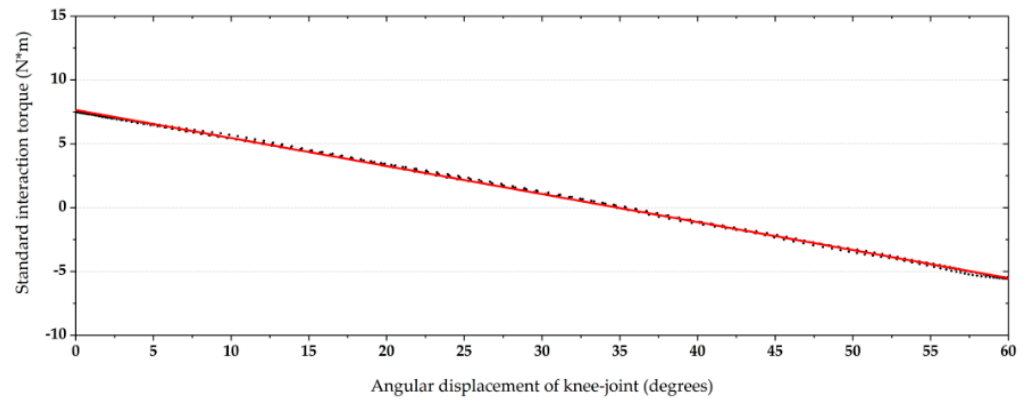

Figure 10. The relationship between the angular displacement of the knee joint and standard interaction torque in the knee joint.

Table 3. Results of the human-exoskeleton interaction measurement experiment.

\begin{tabular}{|c|c|c|}
\hline \multirow{2}{*}{ Subject } & \multicolumn{2}{|c|}{ Linear Equation $y=a x+b$} \\
\hline & a & b \\
\hline H1 (male, 24 years old, weight $/ 60$ kg, height/1.70 m) & -0.21946 & 7.64923 \\
\hline H2 (male, 23 years old weight $/ 65 \mathrm{~kg}$, height $/ 1.75 \mathrm{~m}$ ) & -0.22056 & 8.62145 \\
\hline H3 (female, 25 years old, weight $/ 51 \mathrm{~kg}$, height $/ 1.60 \mathrm{~m}$ ) & -0.21864 & 6.21456 \\
\hline
\end{tabular}

\subsection{Track Tracking Experiments under Different Admittance Conditions}

- No admittance:

Figure 11a is the comparison result of track tracking experiment of subject H1 (male, 24 years old, weight $/ 60 \mathrm{~kg}$, height $/ 1.70 \mathrm{~m}$ ) under different control strategies. It can be seen that the position control accuracy of the ASMVA controller is better than that of GSMC controller. The RMSE (Root mean square error) and PE (Peak error) of track tracking of knee-joint with ASMVA are 0.11 degrees and 0.54 degrees respectively, while those with GSMC are 0.28 degrees and 0.56 degrees respectively. Therefore, the control accuracy of the proposed ASMVA is higher than that of the GSMC. Table 4 summarizes the statistical results of track tracking experiments conducted by three subjects, which shows that the proposed ASMVA control algorithm can ensure the track tracking accuracy of patients' passive training mode. 


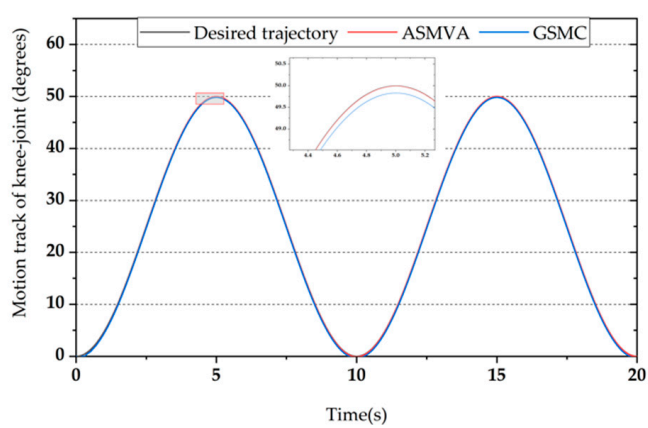

(a)

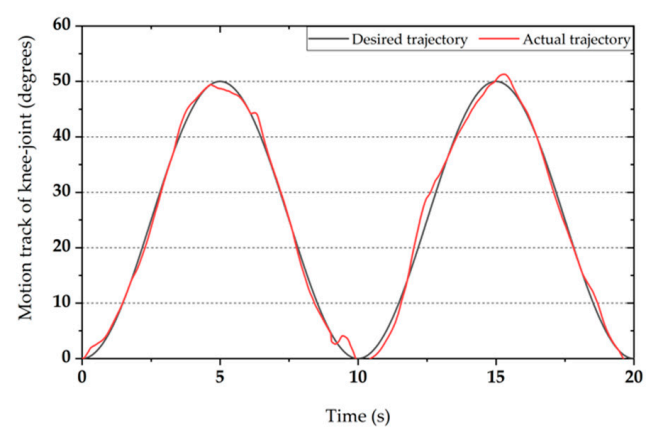

(c)

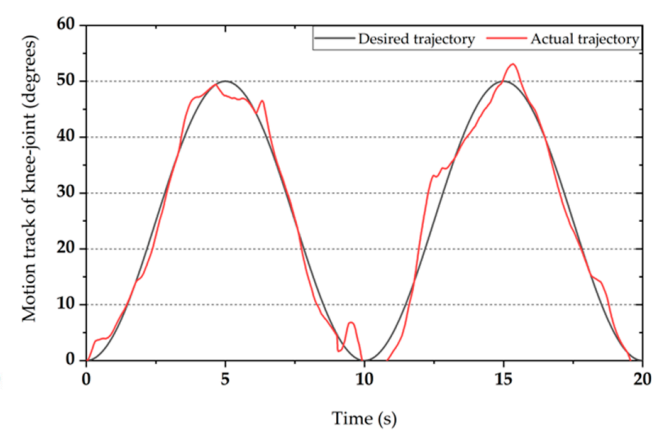

(b)

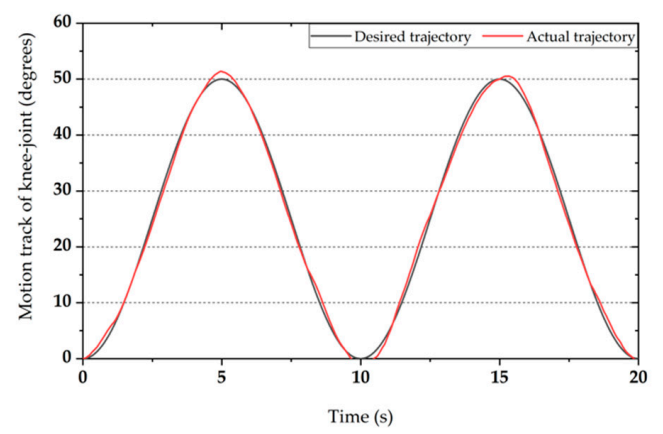

(d)

Figure 11. Experimental results of track tracking under different admittance configurations. (a) Infinite admittance parameters. (b) Small admittance. (c) Medium admittance. (d) Large admittance.

Table 4. Experimental results of track tracking experiment with no admittance.

\begin{tabular}{cccc}
\hline Subject & Controller & RSME/degrees & PE/degrees \\
\hline H1 (male, 24 years old, weight $/ 60 \mathrm{~kg}$, height/1.70 m) & ASMVA & 0.11 & 0.54 \\
& GSMC & 0.28 & 0.56 \\
H2 (male, 23 years old weight $/ 65 \mathrm{~kg}$, height/1.75 m) & ASMVA & 0.11 & 0.65 \\
& GSMC & 0.35 & 0.76 \\
H3 (female, 25 years old, weight $/ 51 \mathrm{~kg}$, height/1.60 m) & ASMVA & 0.10 & 0.57 \\
& GSMC & 0.25 & 0.65 \\
\hline
\end{tabular}

- $\quad$ Fixed admittance:

Figure 11b-d show the track tracking experimental results of subject H1 (male, 24 years old, weight $/ 60 \mathrm{~kg}$, height $/ 1.70 \mathrm{~m}$ ) under the admittance configurations of large admittance, medium admittance, and small admittance, respectively. Table 5 shows the track tracking error under different admittance configurations in condition of fixed admittance. In the small admittance configuration, RMSE is 2.54 degrees, $\mathrm{PE}$ is 3.97 degrees, while in the medium admittance configuration and large admittance configuration, RMSE is 1.27 degrees and 0.88 degrees, PE is 1.99 degrees and 1.31 degrees, respectively. It can be seen that with the increasing of admittance parameters, the deviation between the desired training trajectory and the actual training trajectory will be smaller.

Table 5. Experimental results under different admittance configurations.

\begin{tabular}{ccc}
\hline Admittance Configurations & RSME/degrees & PE/degrees \\
\hline Small admittance & 2.5376 & 3.9702 \\
Medium admittance & 1.2688 & 1.9851 \\
Large admittance & 0.8754 & 1.3143 \\
\hline
\end{tabular}


- Variable admittance:

Figure 12 shows the experimental results of $\mathrm{H} 1$ (male, 24 years old, weight $/ 60 \mathrm{~kg}$, height $/ 1.70 \mathrm{~m}$ ) under the condition of variable admittance. Figure 12a shows the comparison result between measured interaction torque of the knee joint and the estimated active torque of the knee joint. Movement intention of the subject can be obtained according to the estimated active torque of the knee joint, which is shown in Figure 12b. The changes in stiffness and damping of admittance controller during this experiment can be seen in Figure 12c, which indicates that the admittance parameters will change with change in the wearer's active joint torque, and when the active joint torque increases, the admittance parameters will also increase. Figure $12 \mathrm{~d}$ shows the comparison result of the actual and desired trajectory under the condition of variable admittance. Table 6 shows the track tracking error under two kinds of movement intention in the condition of variable admittance, which shows that the track tracking error of the knee joint is small (RSME is 0.69 degrees, PE is 1.65 degrees) when the subject has positive movement intention. The track tracking error is large (RSME is 3.84 degrees, PE is 9.12 degrees) when the subject has negative movement intention. Therefore, the proposed ASMVA controller can recognize the patient's movement intention and adjust the admittance control parameters according to the patient's movement intention. When the patient has positive movement intention, the parameters of admittance controller increase, and the trajectory tracking error of exoskeleton decreases. When the patient has negative movement intention, the parameters of admittance controller will decrease, the track tracking error of exoskeleton will increase, and the interaction between patients and exoskeleton will decrease, so as to protect the patient.

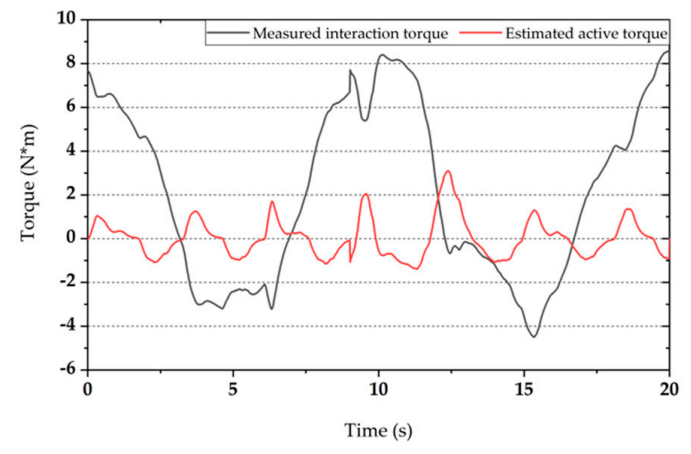

(a)

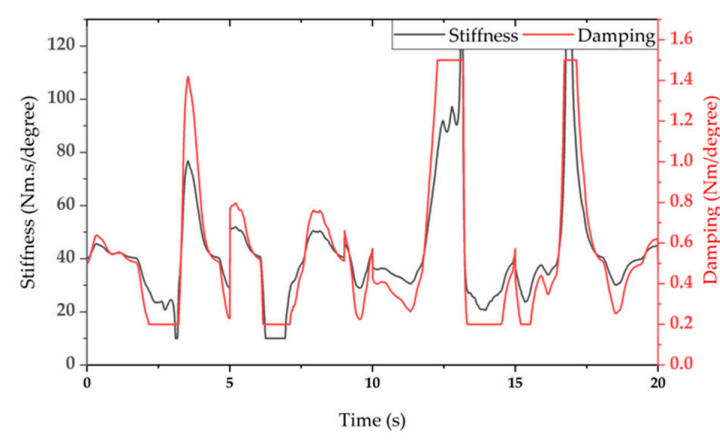

(c)

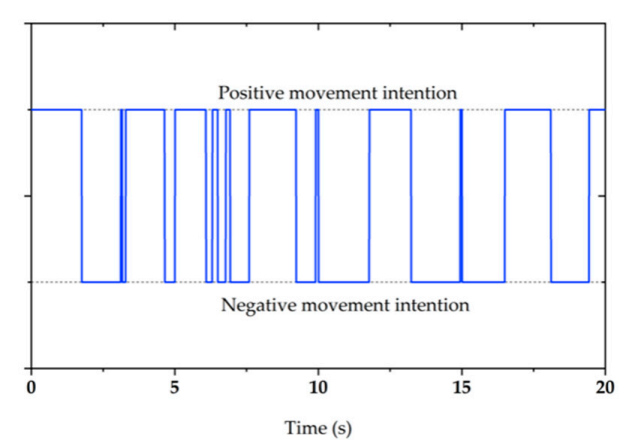

(b)

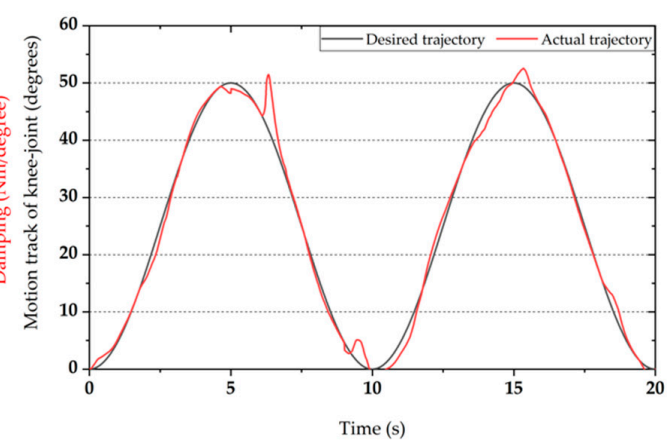

(d)

Figure 12. Experimental results of track tracking under variable admittance conditions. (a) Comparison between measured interaction torque and estimated active torque. (b) Movement intention of subject. (c) Change of admittance parameters. (d) Track tracking result under variable admittance condition. 
Table 6. Experimental results under the condition of variable admittance.

\begin{tabular}{ccc}
\hline Movement Intention & RSME/degrees & PE/degrees \\
\hline Positive & 0.69 & 1.65 \\
Negative & 3.84 & 9.12 \\
\hline
\end{tabular}

\section{Discussion}

This research shows that the ASMVA controller is more suitable for patients with residual muscle strength of lower limbs than the fixed parameter admittance controller, because the ASMVA controller can estimate the patient's active joint torque according to the human-exoskeleton interaction force, so as to obtain the patient's movement intention. It can not only increase the training intensity according to the active muscle strength of the patient during the patient's active movement intention to increase the patient's active participation, but also increase the trajectory adjustment amount during the patient's negative movement intention to ensure patient's safety. The present study differs from other studies in two main aspects. Firstly, this paper proposes an innovative method to estimate the active joint torque of the exoskeleton wearer based on the standard human-exoskeleton interaction torque. Secondly, the ASMVA controller is introduced into the control of the lower limb rehabilitation exoskeleton robot, which realizes the exoskeleton admittance control with the change of admittance parameters.

Unlike the previous research [34,35], in our study, the input of admittance controller is the active joint torque of patients, rather than the interaction between the exoskeleton and patients' limbs. Ee used the variable admittance controller, which can adaptively adjust the admittance parameters according to the patient's movement intention during rehabilitation training. The biggest advantage of our research is that our study took into account the patients' positive and negative movement intentions during rehabilitation training, which realizes the control of the exoskeleton based on the patient's movement intention, so as to improve efficiency of the training. When the patient has positive intention in rehabilitation training, increasing admittance parameters means increasing the stiffness and damping of the system; then, the intensity of rehabilitation training is increased to ensure participation of the patients. When the patient has negative intention in rehabilitation training, decreasing admittance parameters means decrease the stiffness and damping of the system, which can ensure the safety and comfort of the patient.

Clinical research has confirmed that the lack of muscle strength in patients with dyskinesia is an important factor affecting the recovery of limb function [36], and the lack of muscle strength has a direct responsibility in causing sports reinjury [37]. Therefore, in rehabilitation training, it is very important to improve muscle strength of the lower limbs of patients. Research by Krishnan et al. [38] confirmed that improving the participation of patients in the process of rehabilitation training is very helpful in improving the muscle strength of patients. As an example, for patients with locomotive syndrome and sarcopenia, their affected limbs retain a certain of muscle strength. At this time, the ASMVA controller can be applied to the rehabilitation training of this kind of patients, because the ASMVA controller can estimate the active joint torque of the lower limbs of the patients, so as to judge the movement intention of the patients to adjust the rehabilitation training trajectory adaptively. The ASMVA controller can adaptively change the intensity of rehabilitation training by judging the positive movement intention of patients and the strength of muscles, so as to ensure the active participation of patients during the rehabilitation training. Besides, the ASMVA also provides a security mechanism, that is, when the controller detects that the direction of the patient's active joint torque is not the same as that of exoskeleton joint velocity, it reduces the admittance parameters to decrease the system stiffness, thus reducing the interaction between the patient and the exoskeleton, and ensuring the safety of patients during the rehabilitation training.

Finally, it should be noted that the study had one limitation. The basic stiffness and damping of the variable admittance controller need to be adjusted according to the degree of symptoms or recovery degree of muscle strength of lower limb in dyskinesia patients with residual muscle strength. 
Therefore, future work will further improve the robot-assisted patient training evaluation system based on this research, and provide the basis for optimizing system control parameters and rehabilitation training strategies.

\section{Conclusions}

This paper presented a human-exoskeleton coordinated control strategy based on adaptive sliding mode variable admittance control method for a lower limb rehabilitation exoskeleton robot, which could estimate the patient's active joint torque based on human-exoskeleton interaction force, adjust the control parameters of the admittance controller adaptively according to the patient's movement intention, and increase the flexibility of interaction and active participation of the patients. The derivation process of the adaptive sliding mode variable admittance control algorithm was analyzed, and the stability of the system was proved according to the Lyapunov stability criterion. Three healthy volunteers engaged in further experimental studies, including trajectory tracking experiments with no admittance, fixed admittance, and variable admittance adjustment. The experimental results showed that the proposed ASMVA controller had higher position control accuracy than the traditional sliding mode controller. In addition, the proposed ASMVA controller was able to obtain the patient's movement intention according to the patient's active joint torque and adjust the admittance parameters adaptively; this not only increased the training intensity according to the active muscle strength of the patient during active movement intention (to increase active participation), but also increased the trajectory adjustment amount during the patient's negative movement intention to ensure safety.

Author Contributions: Conceptualization, Y.T. and A.Z.; methodology, J.S.; software, Y.T. and H.S.; validation, Y.T., Z.S., and J.S.; writing—original draft preparation, Y.T.; writing-review and editing, A.Z.; visualization, J.S.; supervision, A.Z.; project administration, A.Z., X.Z., and G.C. All authors have read and agreed to the published version of the manuscript.

Funding: This research was funded by the National Key Research and Development Program for Intelligent Robots of the Ministry of Science and Technology, Grant Number 2017YFB1300505, Shenzhen joint key fund project of the national natural fund, Grant Number U1813212.

Conflicts of Interest: The authors declare no conflict of interest.

\section{References}

1. Shi, D.; Zhang, W.; Zhang, W.; Ding, X. A review on lower limb rehabilitation exoskeleton robots. Chin. J. Mech. Eng. 2019, 32, 74. [CrossRef]

2. Jiang, J.G.; Ma, X.F.; Huo, B.; Zhang, Y.D.; Yu, X.Y. Recent advances on lower limb exoskeleton rehabilitation robot. Recent Pat. Eng. 2017, 11, 194-207. [CrossRef]

3. Jezernik, S.; Colombo, G.; Morari, M. Automatic gait-pattern adaptation algorithms for rehabilitation with a 4-dof robotic orthosis. IEEE Trans. Robotic. Autom. 2004, 20, 574-582. [CrossRef]

4. Banala, S.K.; Kim, S.H.; Agrawal, S.K.; Scholz, J.P. Robot assisted gait training with active leg exoskeleton (ALEX). IEEE Trans. Neural Syst. Rehabil. Eng. 2008. [CrossRef]

5. Reinkensmeyer, D.J.; Emken, J.L.; Cramer, S.C. Robotics, motor learning, and neurologic recovery. Annu. Rev. Biomed. Eng. 2004, 6, 497-525. [CrossRef]

6. Marchal-Crespo, L.; Reinkensmeyer, D.J. Review of control strategies for robotic movement training after neurologic injury. J. Neuroeng. Rehabil. 2009, 6, 20. [CrossRef]

7. Bohannon, R.W. Muscle strength and muscle training after stroke. J. Rehabil. Med. 2007, 39, 14-20. [CrossRef]

8. Wang, L.; Wang, S.; van Asseldonk, E.H.; van der Kooij, H. Actively controlled lateral gait assistance in a lower limb exoskeleton. In Proceedings of the 2013 IEEE/RSJ International Conference on Intelligent Robots and Systems, Tokyo, Japan, 3-7 November 2013; pp. 965-970.

9. Sanz-Merodio, D.; Cestari, M.; Arevalo, J.C.; Garcia, E. A lower-limb exoskeleton for gait assistance in quadriplegia. In Proceedings of the 2012 IEEE International Conference on Robotics and Biomimetics (ROBIO), Guangzhou, China, 11-14 December 2012; pp. 122-127. 
10. Talaty, M.; Esquenazi, A.; Briceno, J.E. Differentiating ability in users of the ReWalk TM powered exoskeleton: An analysis of walking kinematics. In Proceedings of the 2013 IEEE 13th International Conference on Rehabilitation Robotics (ICORR), Seattle, WA, USA, 24-26 June 2013; pp. 1-5.

11. Strausser, K.A.; Kazerooni, H. The development and testing of a human machine interface for a mobile medical exoskeleton. In Proceedings of the 2011 IEEE/RSJ International Conference on Intelligent Robots and Systems, San Francisco, CA, USA, 25-30 September 2011; pp. 4911-4916.

12. Iandolo, R.; Marini, F.; Semprini, M.; Laffranchi, M.; Mugnosso, M.; Cherif, A.; Zenzeri, J. Perspectives and Challenges in Robotic Neurorehabilitation. Appl. Sci. 2019, 9, 3183. [CrossRef]

13. Kim, C.Y.; Lee, J.S.; Kim, H.D.; Kim, J.S. The effect of progressive task-oriented training on a supplementary tilt table on lower extremity muscle strength and gait recovery in patients with hemiplegic stroke. Gait Posture 2015, 41, 425-430. [CrossRef]

14. Duschau-Wicke, A.; von Zitzewitz, J.; Caprez, A.; Lunenburger, L.; Riener, R. Path control: A method for patient-cooperative robot-aided gait rehabilitation. IEEE Trans. Neural Syst. Rehabil. Eng. 2009, 18, 38-48. [CrossRef]

15. Liu, J.; Zhang, Y.; Wang, J.; Chen, W. Adaptive sliding mode control for a lower-limb exoskeleton rehabilitation robot. In Proceedings of the 2018 13th IEEE Conference on Industrial Electronics and Applications (ICIEA), Wuhan, China, 31 May-2 June 2018; pp. 1481-1486.

16. Brahmi, B.; Saad, M.; Ochoa-Luna, C.; Rahman, M.H.; Brahmi, A. Adaptive tracking control of an exoskeleton robot with uncertain dynamics based on estimated time-delay control. IEEE/ASME Trans. Mech. 2018, 23, 575-585. [CrossRef]

17. He, W.; Li, Z.; Dong, Y.; Zhao, T. Design and adaptive control for an upper limb robotic exoskeleton in presence of input saturation. IEEE Trans. Neural Netw. Learn. Syst. 2018, 30, 97-108. [CrossRef]

18. Knaepen, K.; Beyl, P.; Duerinck, S.; Hagman, F.; Lefeber, D.; Meeusen, R. Human-robot interaction: Kinematics and muscle activity inside a powered compliant knee exoskeleton. IEEE Trans. Neural Syst. Rehabil. Eng. 2014, 22, 1128-1137. [CrossRef] [PubMed]

19. Kim, S.; Bae, J. Development of a lower extremity exoskeleton system for human-robot interaction. In Proceedings of the 2014 11th International Conference on Ubiquitous Robots and Ambient Intelligence (URAI), Kuala Lumpur, Malaysia, 12-15 November 2014; pp. 132-135.

20. Long, Y.; Du, Z.; Cong, L.; Wang, W.; Zhang, Z.; Dong, W. Active disturbance rejection control based human gait tracking for lower extremity rehabilitation exoskeleton. ISA Trans. 2017, 67, 389-397. [CrossRef] [PubMed]

21. Ergin, M.A.; Patoglu, V. A self-adjusting knee exoskeleton for robot-assisted treatment of knee injuries. In Proceedings of the 2011 IEEE/RSJ International Conference on Intelligent Robots and Systems, San Francisco, CA, USA, 25-30 September 2011; pp. 4917-4922.

22. Bowyer, S.A.; y Baena, F.R. Dissipative control for physical human-Robot interaction. IEEE Trans. Robot. 2015, 31, 1281-1293. [CrossRef]

23. Wu, Q.; Wang, X.; Chen, B.; Wu, H. Development of a minimal-intervention-based admittance control strategy for upper extremity rehabilitation exoskeleton. IEEE Trans. Syst. Man Cybern. 2017, 48, 1005-1016. [CrossRef]

24. Okunev, V.; Nierhoff, T.; Hirche, S. Human-preference-based control design: Adaptive robot admittance control for physical human-robot interaction. In Proceedings of the 2012 IEEE RO-MAN: The 21st IEEE International Symposium on Robot and Human Interactive Communication, Paris, France, 9-13 September 2012; pp. 443-448.

25. Ficuciello, F.; Villani, L.; Siciliano, B. Variable impedance control of redundant manipulators for intuitive human-robot physical interaction. IEEE Trans. Robot. 2015, 31, 850-863. [CrossRef]

26. Yu, W.; Rosen, J.; Li, X. PID admittance control for an upper limb exoskeleton. In Proceedings of the 2011 American Control Conference, San Francisco, CA, USA, 29 June-1 July 2011; pp. 1124-1129.

27. Aydin, Y.; Tokatli, O.; Patoglu, V.; Basdogan, C. Stable physical human-robot interaction using fractional order admittance control. IEEE Trans. Haptics 2018, 11, 464-475. [CrossRef]

28. Pan, L.; Song, A.; Xu, G.; Li, H.; Zeng, H.; Xu, B. Safety supervisory strategy for an upper-limb rehabilitation robot based on impedance control. Int. J. Adv. Robot. Syst. 2013, 10, 127. [CrossRef]

29. Pan, L.; Song, A.; Xu, G.; Li, H.; Xu, B.; Xiong, P. Hierarchical safety supervisory control strategy for robot-assisted rehabilitation exercise. Robotica 2013, 31, 757-766. [CrossRef] 
30. Aguirre-Ollinger, G.; Colgate, J.E.; Peshkin, M.A.; Goswami, A. Design of an active one-degree-of-freedom lower-limb exoskeleton with inertia compensation. Int. J. Robot. Res. 2011, 30, 486-499. [CrossRef]

31. Aguirre-Ollinger, G.; Colgate, J.E.; Peshkin, M.A.; Goswami, A. A 1-DOF assistive exoskeleton with virtual negative damping: Effects on the kinematic response of the lower limbs. In Proceedings of the 2007 IEEE/RSJ International Conference on Intelligent Robots and Systems, San Diego, CA, USA, 29 October-2 November 2007; pp. 1938-1944.

32. Wang, D.; Li, J.; Li, C. An adaptive haptic interaction architecture for knee rehabilitation robot. In Proceedings of the 2009 International Conference on Mechatronics and Automation, Changchun, China, 9-12 August 2009; pp. 84-89.

33. Morbi, A.; Ahmadi, M.; Chan, A.D.; Langlois, R. Stability-guaranteed assist-as-needed controller for powered orthoses. IEEE Trans. Control Syst. Technol. 2013, 22, 745-752. [CrossRef]

34. Han, Y.; Zhu, S.; Zhou, Y.; Gao, H. An admittance controller based on assistive torque estimation for a rehabilitation leg exoskeleton. Intell. Serv. Robot. 2019, 12, 381-391. [CrossRef]

35. Wu, Q.; Wang, X.; Chen, B.; Wu, H. Design and fuzzy sliding mode admittance control of a soft wearable exoskeleton for elbow rehabilitation. IEEE Access 2018, 6, 60249-60263. [CrossRef]

36. Maria, K.C.; Eng, J.J. The relationship of lower-extremity muscle torque to locomotor performance in people with stroke. Phys. Ther. 2003, 83, 49-57.

37. Canning, C.G.; Ada, L.; O'Dwyer, N.J. Abnormal muscle activation characteristics associated with loss of dexterity after stroke. J. Neurol. Sci. 2000, 176, 45-56. [CrossRef]

38. Krishnan, C.; Ranganathan, R.; Dhaher, Y.Y.; Rymer, W.Z. A pilot study on the feasibility of robot-aided leg motor training to facilitate active participation. PLoS ONE 2013, 8, e77370. [CrossRef]

(C) 2020 by the authors. Licensee MDPI, Basel, Switzerland. This article is an open access article distributed under the terms and conditions of the Creative Commons Attribution (CC BY) license (http://creativecommons.org/licenses/by/4.0/). 